\title{
Tevatron Constraints on Models of the Higgs Boson with Exotic Spin and Parity Using Decays to Bottom-Antibottom Quark Pairs
}

T. Aaltonen, ${ }^{21, \dagger}$ V. M. Abazov, ${ }^{13, \sharp}$ B. Abbott,${ }^{116, \$}$ B. S. Acharya, ${ }^{80,}$ M. Adams, ${ }^{98, \ddagger}$ T. Adams, ${ }^{97, \sharp}$ J. P. Agnew, ${ }^{94, \$}$ G. D. Alexeev, ${ }^{13, \dagger}$ G. Alkhazov, ${ }^{88,}$ A. Alton, ${ }^{31, a, \sharp}$ S. Amerio, ${ }^{39 a, 39 b, \dagger}$ D. Amidei ${ }^{31, \dagger}$ A. Anastassov, ${ }^{15, b, \dagger}$ A. Annovi, ${ }^{17, \uparrow}$ J. Antos, ${ }^{12, \dagger}$ G. Apollinari, ${ }^{15, \dagger}$ J. A. Appel, ${ }^{15, \dagger}$ T. Arisawa, ${ }^{52, \dagger}$ A. Artikov, ${ }^{13, \dagger}$ J. Asaadi, ${ }^{47, \dagger}$ W. Ashmanskas, ${ }^{15, \dagger}$ A. Askew,${ }^{97, \dagger}$ S. Atkins, ${ }^{106, \sharp}$ B. Auerbach, ${ }^{2, \dagger}$ K. Augsten, ${ }^{62, \sharp}$ A. Aurisano,${ }^{47, \dagger}$ C. Avila, ${ }^{60, \ddagger}$ F. Azfar, ${ }^{38, \dagger}$ F. Badaud, ${ }^{65, \sharp}$ W. Badgett,,${ }^{15, \dagger}$ T. Bae ${ }^{25, \dagger}$ L. Bagby, ${ }^{15, \$}$ B. Baldin, ${ }^{15, \$}$ D. V. Bandurin, ${ }^{12, *}$ S. Banerjee, ${ }^{80,}$ A. Barbaro-Galtieri, ${ }^{26, \dagger}$ E. Barberis, ${ }^{107, \$}$ P. Baringer, ${ }^{105, \$}$ V. E. Barnes, ${ }^{43, \dagger}$ B. A. Barnett, ${ }^{23, \dagger}$ P. Barria, ${ }^{41,41 \mathrm{c}, \dagger}$ J. F. Bartlett, ${ }^{15, \ddagger}$ P. Bartos, ${ }^{12, \dagger}$ U. Bassler, ${ }^{70, \$}$

M. Bauce ${ }^{39 a, 39 b, \dagger}$ V. Bazterra, ${ }^{98, \$}$ A. Bean, ${ }^{105, \$}$ F. Bedeschi, ${ }^{41 a, \dagger}$ M. Begalli, ${ }^{57, \sharp}$ S. Behari, ${ }^{15, \dagger}$ L. Bellantoni, ${ }^{15, \$}$ G. Bellettini, ${ }^{4 \mathrm{a}, 41 \mathrm{~b}, \dagger}$ J. Bellinger, ${ }^{54, \dagger}$ D. Benjamin, ${ }^{14, \dagger}$ A. Beretvas, ${ }^{15, \dagger}$ S. B. Beri, ${ }^{78, \ddagger}$ G. Bernardi, ${ }^{69, \$}$ R. Bernhard, ${ }^{74, \$}$ I. Bertram, ${ }^{92, \$}$ M. Besançon, ${ }^{70, \$}$ R. Beuselinck, ${ }^{93,}$ P. C. Bhat, ${ }^{15,}$ S. Bhatia, ${ }^{108, \ddagger}$ V. Bhatnagar, ${ }^{78, \$}$ A. Bhatti, ${ }^{45, \dagger}$ K. R. Bland ${ }^{5,}$ G. Blazey, ${ }^{99,}$ S. Blessing, ${ }^{97,}$ K. Bloom, ${ }^{109, \star}$ B. Blumenfeld,${ }^{23, \dagger}$ A. Bocci, ${ }^{14,}$ A. Bodek ${ }^{44, \dagger}$ A. Boehnlein, ${ }^{15,}$ D. Boline, ${ }^{13,}$

E. E. Boos, ${ }^{86, *}$ G. Borissov, ${ }^{92,}$ D. Bortoletto, ${ }^{43, \uparrow}$ M. Borysova, ${ }^{91, c, \$}$ J. Boudreau, ${ }^{42, \uparrow}$ A. Boveia, ${ }^{11, \dagger}$ A. Brandt, ${ }^{119, \$}$

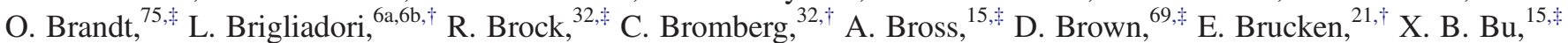
J. Budagov, ${ }^{13, \dagger}$ H. S. Budd, ${ }^{44, \uparrow}$ M. Buehler, ${ }^{15, \$}$ V. Buescher ${ }^{76, \sharp}$ V. Bunichev, ${ }^{86, \$}$ S. Burdin, ${ }^{92, d, \$}$ K. Burkett, ${ }^{15, \dagger}$ G. Busetto, ${ }^{39 a, 39 b, \dagger}$ P. Bussey, ${ }^{19, \uparrow}$ C. P. Buszello, ${ }^{90, \$}$ P. Butti,${ }^{41 a, 41 b, \dagger}$ A. Buzatu, ${ }^{19, \dagger}$ A. Calamba, ${ }^{10, \dagger}$ E. Camacho-Pérez ${ }^{83, \$}$ S. Camarda, ${ }^{4, \dagger}$ M. Campanelli, ${ }^{28, \uparrow}$ F. Canelli, ${ }^{11, e, \dagger}$ B. Carls,${ }^{22, \dagger}$ D. Carlsmith,,${ }^{54, \dagger}$ R. Carosi, ${ }^{41 \mathrm{a}, \uparrow}$ S. Carrillo, ${ }^{16, f, \dagger}$ B. Casal,${ }^{9, \mathrm{~g}, \dagger}$ M. Casarsa, ${ }^{48, \dagger}$ B. C. K. Casey, ${ }^{15,}$ H. Castilla-Valdez, ${ }^{83, \$}$ A. Castro, ${ }^{6 a, 6 b, \dagger}$ P. Catastini,${ }^{20, \dagger}$ S. Caughron,,${ }^{32, \$}$ D. Cauz ${ }^{48 \mathrm{a}, 48 \mathrm{~b}, \mathrm{a} 48 \mathrm{c}, \uparrow}$ V. Cavaliere, ${ }^{22, \dagger}$ A. Cerri,${ }^{26, \mathrm{~h}, \dagger}$ L. Cerrito, ${ }^{28, \mathrm{i}, \uparrow}$ S. Chakrabarti, ${ }^{113, \$}$ K. M. Chan, ${ }^{103, \sharp}$ A. Chandra, ${ }^{121, \$}$ E. Chapon, ${ }^{70, *}$ G. Chen, ${ }^{105, \$}$ Y. C. Chen, ${ }^{1, \dagger}$ M. Chertok, ${ }^{7, \dagger}$ G. Chiarelli, ${ }^{41 a, \uparrow}$ G. Chlachidze, ${ }^{15, \dagger}$ K. Cho, ${ }^{25, \dagger}$ S. W. Cho, ${ }^{82, \sharp}$ S. Choi,${ }^{82, \$}$ D. Chokheli, ${ }^{1, \dagger}$ B. Choudhary, ${ }^{79, \dagger}$ S. Cihangir, ${ }^{15, \sharp}$ D. Claes, ${ }^{109, \sharp}$ A. Clark, ${ }^{18, \dagger}$ C. Clarke, ${ }^{53, \dagger}$ J. Clutter, ${ }^{105, \$}$ M. E. Convery, ${ }^{15, \dagger}$ J. Conway, ${ }^{7, \dagger}$ M. Cooke, ${ }^{15, j,}$ W. E. Cooper, ${ }^{15, \dagger}$ M. Corbo,,${ }^{15, k, \dagger}$ M. Corcoran, ${ }^{121, \$}$ M. Cordelli, ${ }^{17, \dagger}$

F. Couderc, ${ }^{70, \$}$ M.-C. Cousinou, ${ }^{67, \$}$ C. A. Cox,${ }^{7, \dagger}$ D. J. Cox,${ }^{7, \dagger}$ M. Cremonesi,${ }^{41 a, \dagger}$ D. Cruz, ${ }^{47, \dagger}$ J. Cuevas,,${ }^{9,1} \dagger$

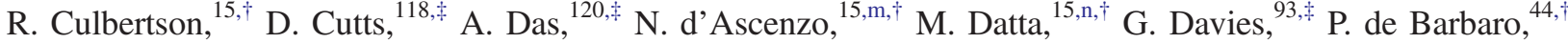
S. J. de Jong, ${ }^{84,85, \$}$ E. De La Cruz-Burelo, ${ }^{83, \$}$ F. Déliot, ${ }^{70, \$}$ R. Demina,${ }^{44, \$}$ L. Demortier, ${ }^{45, \dagger}$ M. Deninno, ${ }^{6 a, \dagger}$ D. Denisov, ${ }^{15,}$

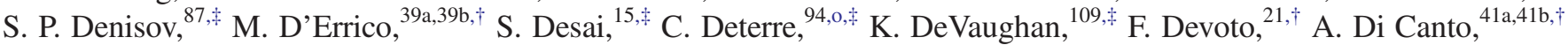
B. Di Ruzza, ${ }^{15, p, \dagger}$ H. T. Diehl, ${ }^{15,}$ M. Diesburg, ${ }^{15, \$}$ P. F. Ding, ${ }^{94, \$}$ J. R. Dittmann, ${ }^{5, \dagger}$ A. Dominguez, ${ }^{109, \$}$ S. Donati, ${ }^{41 a, 41 b, \uparrow}$ M. D’Onofrio, ${ }^{27, \dagger}$ M. Dorigo, ${ }^{48 a, 48 d, \uparrow}$ A. Driutti, ${ }^{48 a, 48 b, a 48 c, \uparrow}$ A. Dubey, ${ }^{79, \$}$ L. V. Dudko, ${ }^{86, \$}$ A. Duperrin, ${ }^{67, \$}$ S. Duttt, ${ }^{78, \$}$ M. Eads, ${ }^{99, *}$ K. Ebina ${ }^{52, \dagger}$ R. Edgar ${ }^{31, \dagger}$ D. Edmunds, ${ }^{32,+}$ A. Elagin, ${ }^{47, \dagger}$ J. Ellison, ${ }^{96,+}$ V. D. Elvira, ${ }^{15, *}$ Y. Enari, ${ }^{69, *}$ R. Erbacher, ${ }^{7, \dagger}$ S. Errede, ${ }^{22, \dagger}$ B. Esham, ${ }^{22, \dagger}$ H. Evans, ${ }^{101, \$}$ V. N. Evdokimov, ${ }^{87, \$}$ S. Farrington, ${ }^{38, \dagger}$ A. Fauré, ${ }^{70, \$}$ L. Feng, ${ }^{99, \$}$ T. Ferbel, ${ }^{44, \ddagger}$ J. P. Fernández Ramos,${ }^{29, \dagger}$ F. Fiedler ${ }^{76, \$}$ R. Field, ${ }^{16, \dagger}$ F. Filthaut, ${ }^{84,85, \$}$ W. Fisher, ${ }^{32,}$ H. E. Fisk, ${ }^{15,}$ G. Flanagan, ${ }^{15, q, \dagger}$ R. Forrest ${ }^{7, \dagger}$ M. Fortner, ${ }^{99, \ddagger}$ H. Fox,${ }^{92, \ddagger}$ M. Franklin, ${ }^{20, \dagger}$ J. C. Freeman, ${ }^{15, \dagger}$ H. Frisch, ${ }^{11, \dagger}$ S. Fuess, ${ }^{15,}$ Y. Funakoshi, ${ }^{52, \uparrow}$ C. Galloni, ${ }^{41 a, 41 b, \dagger}$ P. H. Garbincius, ${ }^{15, \$}$ A. Garcia-Bellido, ${ }^{44, \$}$ J. A. García-González, ${ }^{83, \$}$ A. F. Garfinkel, ${ }^{43, \dagger}$ P. Garosi ${ }^{41 \mathrm{a}, 41 \mathrm{c}, \dagger}$ V. Gavrilov, ${ }^{33,}$ W. Geng, ${ }^{67,32, \sharp}$ C. E. Gerber, ${ }^{98, *}$ H. Gerberich, ${ }^{22, \uparrow}$ E. Gerchtein, ${ }^{15, \dagger}$ Y. Gershtein, ${ }^{110, *}$ S. Giagu, ${ }^{46 a, \uparrow}$ V. Giakoumopoulou, ${ }^{3, \dagger}$ K. Gibson, ${ }^{42, \uparrow}$ C. M. Ginsburg, ${ }^{15, \dagger}$ G. Ginther, ${ }^{15,44, \uparrow}$ N. Giokaris, ${ }^{3, \dagger}$ P. Giromini, ${ }^{17, \uparrow}$ V. Glagolev ${ }^{13, \dagger}$ D. Glenzinski, ${ }^{15, \dagger}$ O. Gogota, ${ }^{91, \$}$ M. Gold ${ }^{34, \dagger}$ D. Goldin, ${ }^{47, \dagger}$ A. Golossanov, ${ }^{15, \dagger}$ G. Golovanov, ${ }^{13, ;}$ G. Gomez,${ }^{9, \dagger}$ G. Gomez-Ceballos, ${ }^{30, \dagger}$ M. Goncharov,${ }^{30, \uparrow}$ O. González López, ${ }^{29, \dagger}$ I. Gorelov, ${ }^{34, \dagger}$ A. T. Goshaw, ${ }^{14, \dagger}$

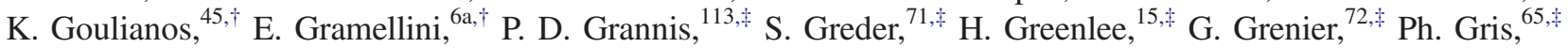
J.-F. Grivaz, ${ }^{68, \sharp}$ A. Grohsjean, ${ }^{70,0, \$}$ C. Grosso-Pilcher, ${ }^{1, \dagger}$ R. C. Group, ${ }^{51,15, \dagger}$ S. Grünendahl, ${ }^{15, *}$ M. W. Grünewald, ${ }^{81, *}$ T. Guillemin, ${ }^{68, \sharp}$ J. Guimaraes da Costa, ${ }^{20, \dagger}$ G. Gutierrez, ${ }^{15, \star}$ P. Gutierrez, ${ }^{116, \sharp}$ S. R. Hahn, ${ }^{15, \dagger}$ J. Haley, ${ }^{117, \sharp}$ J. Y. Han, ${ }^{44, \dagger}$ L. Han, ${ }^{59, \$}$ F. Happacher, ${ }^{17, \dagger}$ K. Hara, ${ }^{49, \uparrow}$ K. Harder, ${ }^{94, \ddagger}$ M. Hare, ${ }^{50, \uparrow}$ A. Harel, ${ }^{44, \dagger}$ R. F. Harr, ${ }^{53, \uparrow}$ T. Harrington-Taber, ${ }^{15, r, \uparrow}$ K. Hatakeyama ${ }^{5, \dagger}$ J. M. Hauptman, ${ }^{104, \$}$ C. Hays, ${ }^{38, \dagger}$ J. Hays, ${ }^{93, \$}$ T. Head,${ }^{94, \$}$ T. Hebbeker, ${ }^{73, \sharp}$ D. Hedin, ${ }^{99, \$}$ H. Hegab, ${ }^{17, \sharp}$ J. Heinrich, ${ }^{40, \uparrow}$ A. P. Heinson, ${ }^{96, \$}$ U. Heintz, ${ }^{118, \ddagger}$ C. Hensel,${ }^{56, \$}$ I. Heredia-De La Cruz ${ }^{83, s, \$}$ M. Herndon, ${ }^{54, \dagger}$ K. Herner, ${ }^{15, \$}$ G. Hesketh, ${ }^{94, t, \ddagger}$ M. D. Hildreth, ${ }^{103, \ddagger}$ R. Hirosky, ${ }^{122, \$}$ T. Hoang, ${ }^{97, \ddagger}$ J. D. Hobbs, ${ }^{11, *}$ A. Hocker, ${ }^{15, \dagger}$ B. Hoeneisen, ${ }^{64, \hbar}$ J. Hogan, ${ }^{121, \$}$ M. Hohlfeld, ${ }^{76, \$}$ J. L. Holzbauer, ${ }^{108, \$}$ Z. Hong,${ }^{47, \dagger}$ W. Hopkins, ${ }^{15, u, \uparrow}$ S. Hou, ${ }^{1, \dagger}$ I. Howley, ${ }^{119, \star}$ Z. Hubacek,${ }^{62,70, \$}$

R. E. Hughes,${ }^{35, \dagger}$ U. Husemann,${ }^{55, \dagger}$ M. Hussein,${ }^{32, v, \uparrow}$ J. Huston, ${ }^{32, \dagger}$ V. Hynek, ${ }^{62, \$}$ I. Iashvili, ${ }^{12, \$}$ Y. Ilchenko, ${ }^{120, \$}$

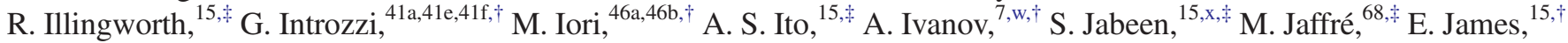

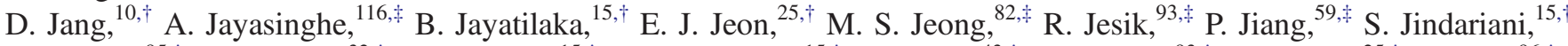
K. Johns, ${ }^{95, \$}$ E. Johnson, ${ }^{32, \$}$ M. Johnson, ${ }^{15,}$ A. Jonckheere, ${ }^{15,}$ M. Jones, ${ }^{43, \dagger}$ P. Jonsson, ${ }^{93, *}$ K. K. Joo, ${ }^{25, \dagger}$ J. Joshi, ${ }^{96, \$}$ 
S. Y. Jun, ${ }^{10, \dagger}$ A. W. Jung, ${ }^{15,}$ T. R. Junk,${ }^{15, \dagger}$ A. Juste, ${ }^{89, \$}$ E. Kajfasz, ${ }^{67,}$ M. Kambeitz, ${ }^{24, \dagger}$ T. Kamon, ${ }^{25,47, \dagger}$ P. E. Karchin, ${ }^{53, \dagger}$ D. Karmanov ${ }^{86, *}$ A. Kasmi ${ }^{5, \dagger}$ Y. Kato, ${ }^{37, y, \dagger}$ I. Katsanos, ${ }^{109, \$}$ M. Kaur, ${ }^{78, \$}$ R. Kehoe, ${ }^{120, \$}$ S. Kermiche, ${ }^{67, \$}$ W. Ketchum, ${ }^{11, z, \dagger}$ J. Keung, ${ }^{40, \dagger}$ N. Khalatyan, ${ }^{15, \$}$ A. Khanov, ${ }^{117, \$}$ A. Kharchilava, ${ }^{12, \$}$ Y. N. Kharzheev, ${ }^{13, \ddagger}$ B. Kilminster, ${ }^{15, e, \dagger}$ D. H. Kim,${ }^{25, \dagger}$ H. S. Kim,${ }^{25, \dagger}$ J. E. Kim, ${ }^{25, \dagger}$ M. J. Kim, ${ }^{17, \dagger}$ S. H. Kim,${ }^{49, \dagger}$ S. B. Kim, ${ }^{25, \uparrow}$ Y. J. Kim, ${ }^{25, \dagger}$ Y. K. Kim, ${ }^{11, \dagger}$ N. Kimura, ${ }^{52, \uparrow}$ M. Kirby, ${ }^{15, \dagger}$ I. Kiselevich, ${ }^{33, \$}$ K. Knoepfel,${ }^{15, \dagger}$ J. M. Kohli, ${ }^{78, \$}$ K. Kondo, ${ }^{52,{ }^{*}, \dagger}$ D. J. Kong, ${ }^{25, \uparrow}$ J. Konigsberg, ${ }^{16, \dagger}$ A. V. Kotwal, ${ }^{14, \dagger}$ A. V. Kozelov, ${ }^{87, \sharp}$ J. Kraus, ${ }^{108, \ddagger}$ M. Kreps ${ }^{24, \dagger}$ J. Kroll,${ }^{40, \dagger}$ M. Kruse, ${ }^{14, \dagger}$ T. Kuhr, ${ }^{24, \dagger}$ A. Kumar, ${ }^{12, \$}$ A. Kupco, ${ }^{63, \$}$ M. Kurata, ${ }^{49, \dagger}$ T. Kurča, ${ }^{72, \$}$ V. A. Kuzmin, ${ }^{86, \$}$ A. T. Laasanen, ${ }^{43, \dagger}$ S. Lammel, ${ }^{15, \dagger}$ S. Lammers, ${ }^{101, \$}$ M. Lancaster, ${ }^{28, \dagger}$ K. Lannon, ${ }^{35, a a, \dagger}$ G. Latino, ${ }^{41 a, 41 c, \uparrow}$ P. Lebrun, ${ }^{72, \sharp}$ H. S. Lee ${ }^{82, \ddagger}$ H. S. Lee, ${ }^{25, \dagger}$ J. S. Lee, ${ }^{25, \dagger}$ S. W. Lee, ${ }^{104, \$}$

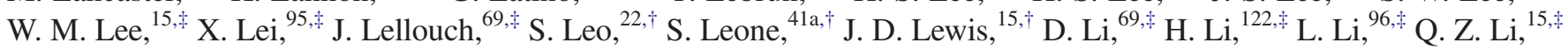
J. K. Lim ${ }^{82, \$}$ A. Limosani, ${ }^{14, b b, \dagger}$ D. Lincoln,${ }^{15,}$ J. Linnemann,${ }^{32, \$}$ V. V. Lipaev, ${ }^{87, \$}$ E. Lipeles, ${ }^{40, \dagger}$ R. Lipton, ${ }^{15, \$}$ A. Lister ${ }^{18, c c, \dagger}$ H. Liu ${ }^{51, \dagger}$ H. Liu, ${ }^{120, \dagger}$ Q. Liu, ${ }^{43, \dagger}$ T. Liu, ${ }^{15, \dagger}$ Y. Liu, ${ }^{59, \$}$ A. Lobodenko, ${ }^{88, \hbar}$ S. Lockwitz, ${ }^{55, \dagger}$ A. Loginov, ${ }^{55, \dagger}$ M. Lokajicek, ${ }^{63,}$ R. Lopes de Sa, ${ }^{15, \$}$ D. Lucchesi, ${ }^{39 a, 39 b, \dagger}$ A. Lucà, ${ }^{17, \dagger}$ J. Lueck, ${ }^{24, \dagger}$ P. Lujan, ${ }^{26, \dagger}$ P. Lukens, ${ }^{15, \dagger}$

R. Luna-Garcia, ${ }^{83, d d, \$}$ G. Lungu, ${ }^{45, \uparrow}$ A. L. Lyon, ${ }^{15, \$}$ J. Lys, ${ }^{26, \uparrow}$ R. Lysak, ${ }^{12, e e, \uparrow}$ A. K. A. Maciel, ${ }^{56, \$}$ R. Madar, ${ }^{74, \$}$ R. Madrak ${ }^{15, \dagger}$ P. Maestro, ${ }^{41 \mathrm{a}, 41 \mathrm{c}, \dagger}$ R. Magaña-Villalba, ${ }^{83, \ddagger}$ S. Malik, ${ }^{45, \dagger}$ S. Malik, ${ }^{109, \$}$ V. L. Malyshev, ${ }^{13, \ddagger}$ G. Manca, ${ }^{27, f f, \dagger}$ A. Manousakis-Katsikakis, ${ }^{3, \dagger}$ J. Mansour, ${ }^{75, \$}$ L. Marchese,${ }^{6 a, g g, \dagger}$ F. Margaroli ${ }^{46 a, \dagger}$ P. Marino, ${ }^{41 a, 41 \mathrm{~d}, \dagger}$ J. Martínez-Ortega ${ }^{83, \sharp}$ K. Matera, ${ }^{22, \dagger}$ M. E. Mattson, ${ }^{53, \dagger}$ A. Mazzacane, ${ }^{15, \dagger}$ P. Mazzanti, ${ }^{6 a, \dagger}$ R. McCarthy, ${ }^{113, \downarrow}$ C. L. McGivern, ${ }^{94, \ddagger}$ R. McNulty, ${ }^{27, \text { hh }, \dagger}$ A. Mehta, ${ }^{27, \dagger}$ P. Mehtala,${ }^{21, \dagger}$ M. M. Meijer, ${ }^{84,85, \ddagger}$ A. Melnitchouk, ${ }^{15, \ddagger}$ D. Menezes, ${ }^{99,}$ P. G. Mercadante, ${ }^{58, \ddagger}$ M. Merkin,${ }^{86, *}$ C. Mesropian, ${ }^{45, \dagger}$ A. Meyer, ${ }^{73, \$}$ J. Meyer, ${ }^{75, i, ;}$ T. Miao, ${ }^{15, \dagger}$ F. Miconi,${ }^{71, \$}$ D. Mietlicki, ${ }^{31, \dagger}$ A. Mitra, ${ }^{1, \dagger}$ H. Miyake, ${ }^{49, \dagger}$ S. Moed ${ }^{15, \uparrow}$ N. Moggi ${ }^{6 a, \uparrow}$ N. K. Mondal,${ }^{80, \ddagger}$ C. S. Moon, ${ }^{15, k, \uparrow}$ R. Moore,${ }^{15, j \mathrm{j}, \mathrm{kk}, \uparrow}$ M. J. Morello, ${ }^{41 \mathrm{a}, 41 \mathrm{~d}, \uparrow}$ A. Mukherjee, ${ }^{15, \uparrow}$ M. Mulhearn, ${ }^{122, \downarrow}$ Th. Muller, ${ }^{24, \dagger}$ P. Murat,${ }^{15, \dagger}$ M. Mussini, ${ }^{6 a, 6 b, \dagger}$ J. Nachtman,${ }^{15, r, \dagger}$ Y. Nagai, ${ }^{49, \dagger}$ J. Naganoma, ${ }^{52, \uparrow}$ E. Nagy, ${ }^{67, \sharp}$ I. Nakano ${ }^{36, \dagger}$ A. Napier, ${ }^{50, \uparrow}$ M. Narain, ${ }^{118, \sharp}$ R. Nayyar, ${ }^{95, \$}$ H. A. Neal, ${ }^{31, \$}$ J. P. Negret, ${ }^{60, \$}$ J. Nett,,${ }^{47, \dagger}$ C. Neu, ${ }^{51, \uparrow}$ P. Neustroev ${ }^{88, \$}$ H. T. Nguyen, ${ }^{122, \$}$ T. Nigmanov, ${ }^{42, \dagger}$ L. Nodulman, ${ }^{2, \dagger}$ S. Y. Noh, ${ }^{25, \dagger}$ O. Norniella, ${ }^{22, \uparrow}$ T. Nunnemann, ${ }^{77, \$}$ L. Oakes ${ }^{38, \uparrow}$ S. H. Oh, ${ }^{14, \uparrow}$ Y. D. Oh ${ }^{25, \dagger}$ I. Oksuzian, ${ }^{51, \dagger}$ T. Okusawa, ${ }^{37, \dagger}$ R. Orava, ${ }^{21, \dagger}$ J. Orduna, ${ }^{121, \sharp}$ L. Ortolan, ${ }^{4, \dagger}$ N. Osman, ${ }^{67, \$}$ J. Osta, ${ }^{103, \$}$ C. Pagliarone, ${ }^{48 a, \dagger}$ A. Pal,${ }^{19, \$}$ E. Palencia, ${ }^{9, h, \dagger}$ P. Palni,,${ }^{34, \dagger}$ V. Papadimitriou, ${ }^{15, \dagger}$ N. Parashar, ${ }^{102, \$}$ V. Parihar, ${ }^{118, \sharp}$ S. K. Park, ${ }^{82, \sharp}$ W. Parker,${ }^{54, \dagger}$ R. Partridge, ${ }^{118,11, \$}$ N. Parua, ${ }^{101, \sharp}$ A. Patwa, ${ }^{114, \mathrm{~mm}, \sharp}$ G. Pauletta, ${ }^{48 \mathrm{a}, 48 \mathrm{~b}, \mathrm{a} 48 \mathrm{c}, \uparrow}$ M. Paulini,${ }^{10, \dagger}$ C. Paus,${ }^{30, \dagger}$ B. Penning,${ }^{15, \$}$ M. Perfilov,${ }^{86, \$}$ Y. Peters,${ }^{94,}$ K. Petridis, ${ }^{94,}$ G. Petrillo, ${ }^{44, \$}$ P. Pétroff, ${ }^{6, \$}$ T. J. Phillips, ${ }^{14, \dagger}$ G. Piacentino, ${ }^{15, \text { nn, }}$ E. Pianori, ${ }^{40, \dagger}$ J. Pilot,${ }^{7, \dagger}$ K. Pitts, ${ }^{22, \dagger}$ C. Plager, ${ }^{8, \dagger}$ M.-A. Pleier, ${ }^{114, \$}$ V. M. Podstavkov, ${ }^{15, \$}$ L. Pondrom, ${ }^{54, \dagger}$ A. V. Popov, ${ }^{87,}$ S. Poprocki, ${ }^{15, u, \dagger}$ K. Potamianos, ${ }^{26, \dagger}$ A. Pranko, ${ }^{26, \dagger}$ M. Prewitt, ${ }^{121, \$}$ D. Price, ${ }^{94, \hbar}$ N. Prokopenko, ${ }^{87, \$}$ F. Prokoshin, ${ }^{13,00, \dagger}$ F. Ptohos, ${ }^{17, p p, \dagger}$ G. Punzi, ${ }^{41 \mathrm{a}, 41 \mathrm{~b}, \uparrow}$ J. Qian, ${ }^{31, \hbar}$ A. Quadt, ${ }^{75, \hbar}$ B. Quinn, ${ }^{108, \sharp}$

P. N. Ratoff, ${ }^{92, \$}$ I. Razumov, ${ }^{87, \$}$ I. Redondo Fernández ${ }^{29, \dagger}$ P. Renton, ${ }^{38, \uparrow}$ M. Rescigno, ${ }^{46 a, \dagger}$ F. Rimondi,,${ }^{6,{ }^{*}, \dagger}$ I. Ripp-Baudot, ${ }^{71, \sharp}$ L. Ristori, ${ }^{41 a, 15,{ }^{\dagger}}$ F. Rizatdinova, ${ }^{117, \sharp}$ A. Robson, ${ }^{19, \dagger}$ T. Rodriguez, ${ }^{40, \dagger}$ S. Rolli, ${ }^{50, q q, \dagger}$ M. Rominsky, ${ }^{15, \$}$ M. Ronzani, ${ }^{41 \mathrm{a}, 41 \mathrm{~b}, \dagger}$ R. Roser, ${ }^{15, \dagger}$ J. L. Rosner, ${ }^{11, \dagger}$ A. Ross, ${ }^{92, \$}$ C. Royon,${ }^{70, \$}$ P. Rubinov, ${ }^{15, \$}$ R. Ruchti, ${ }^{103, \$}$ F. Ruffini, ${ }^{41 a, 41 c, \uparrow}$ A. Ruiz, ${ }^{9, \dagger}$ J. Russ, ${ }^{10, \dagger}$ V. Rusu, ${ }^{15, \dagger}$ G. Sajot,${ }^{66,+}$ W. K. Sakumoto, ${ }^{44, \dagger}$ Y. Sakurai, ${ }^{52, \dagger}$ A. Sánchez-Hernández, ${ }^{83, ;}$

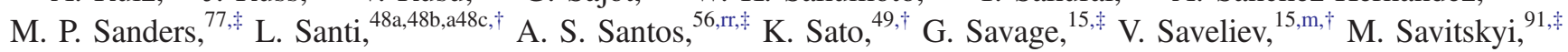

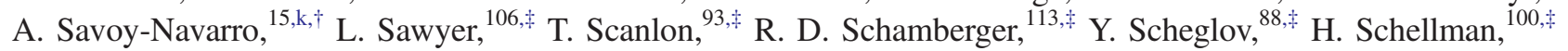
P. Schlabach, ${ }^{15, \dagger}$ E. E. Schmidt,${ }^{15, \dagger}$ C. Schwanenberger, ${ }^{94,}$ T. Schwarz,${ }^{31, \dagger}$ R. Schwienhorst, ${ }^{32, \downarrow}$ L. Scodellaro, ${ }^{9, \dagger}$ F. Scuri, ${ }^{41 a,{ }^{\dagger}}$

S. Seidel,${ }^{34, \dagger}$ Y. Seiya,${ }^{37, \dagger}$ J. Sekaric, ${ }^{105, \dagger}$ A. Semenov, ${ }^{13, \dagger}$ H. Severini, ${ }^{116, \$}$ F. Sforza, ${ }^{41 a, 41 b, \dagger}$ E. Shabalina, ${ }^{75, \$}$

S. Z. Shalhout, ${ }^{7, \dagger}$ V. Shary, ${ }^{70, \star}$ S. Shaw, ${ }^{94, *}$ A. A. Shchukin, ${ }^{87, *}$ T. Shears, ${ }^{27, \dagger}$ P. F. Shepard, ${ }^{42, \dagger}$ M. Shimojima, ${ }^{49, s s, \uparrow}$ M. Shochet ${ }^{11, \dagger}$ I. Shreyber-Tecker, ${ }^{33, \dagger}$ V. Simak ${ }^{62, \$}$ A. Simonenko, ${ }^{13, \dagger}$ P. Skubic, ${ }^{116, \$}$ P. Slattery, ${ }^{44, \hbar}$ K. Sliwa, ${ }^{50, \dagger}$ D. Smirnov, ${ }^{103, \sharp}$ J. R. Smith, ${ }^{7, \dagger}$ F. D. Snider, ${ }^{15,}$ G. R. Snow, ${ }^{109, \sharp}$ J. Snow, ${ }^{115, *}$ S. Snyder, ${ }^{114, \sharp}$ S. Söldner-Rembold, ${ }^{94, \$}$ H. Song, ${ }^{42, \dagger}$ L. Sonnenschein, ${ }^{73,}$ V. Sorin,${ }^{4, \dagger}$ K. Soustruznik, ${ }^{61, *}$ R. St. Denis, ${ }^{19,{ }^{*}, \dagger}$ M. Stancari, ${ }^{15, \dagger}$ J. Stark, ${ }^{66, \$}$ D. Stentz, ${ }^{15, b, \dagger}$ D. A. Stoyanova, ${ }^{87, \sharp}$ M. Strauss, ${ }^{116, \sharp}$ J. Strologas,${ }^{34, \dagger}$ Y. Sudo, ${ }^{49, \dagger}$ A. Sukhanov, ${ }^{15, \dagger}$ I. Suslov, ${ }^{13, \dagger}$ L. Suter, ${ }^{94, \sharp}$ P. Svoisky, ${ }^{116, \sharp}$ K. Takemasa, ${ }^{49, \dagger}$ Y. Takeuchi, ${ }^{49, \dagger}$ J. Tang, ${ }^{11, \uparrow}$ M. Tecchio, ${ }^{31, \uparrow}$ P. K. Teng, ${ }^{1, \dagger}$ J. Thom, ${ }^{15, \mathrm{u}, \uparrow}$ E. Thomson, ${ }^{40, \uparrow}$ V. Thukral, ${ }^{47, \uparrow}$ M. Titov ${ }^{70, \$}$ D. Toback, ${ }^{47, \dagger}$ S. Tokar, ${ }^{12, \uparrow}$ V. V. Tokmenin,${ }^{13, \sharp}$ K. Tollefson,${ }^{32, \dagger}$ T. Tomura, ${ }^{49, \dagger}$ D. Tonelli,,${ }^{15, h, \dagger}$ S. Torre, ${ }^{17, \uparrow}$ D. Torretta, ${ }^{15, \uparrow}$ P. Totaro ${ }^{39 a, \dagger}$ M. Trovato, ${ }^{41 a, 41 d, \dagger}$ Y.-T. Tsai, ${ }^{44, \$}$ D. Tsybychev,${ }^{11, \$}$ B. Tuchming, ${ }^{70, \$}$ C. Tully, ${ }^{11, \$}$

F. Ukegawa, ${ }^{49, \uparrow}$ S. Uozumi,${ }^{25, \dagger}$ L. Uvarov, ${ }^{88, \sharp}$ S. Uvarov, ${ }^{88,}$ S. Uzunyan, ${ }^{99, \$}$ R. Van Kooten, ${ }^{101, \$}$ W. M. van Leeuwen, ${ }^{84, \$}$ N. Varelas, ${ }^{98, \$}$ E. W. Varnes ${ }^{95, \$}$ I. A. Vasilyev, ${ }^{87, \$}$ F. Vázquez,${ }^{16, f, \dagger}$ G. Velev, ${ }^{15, \dagger}$ C. Vellidis, ${ }^{15, \dagger}$ A. Y. Verkheev, ${ }^{13, \$}$ C. Vernieri, ${ }^{41 a, 41 \mathrm{~d}, \dagger}$ L. S. Vertogradov,${ }^{13, \$}$ M. Verzocchi, ${ }^{15, \$}$ M. Vesterinen, ${ }^{94, \$}$ M. Vidal,${ }^{43, \dagger}$ D. Vilanova, ${ }^{70, \$}$ R. Vilar,,${ }^{9, \dagger}$ J. Vizán, ${ }^{9, t, \dagger}$ M. Vogel,${ }^{34, \dagger}$ P. Vokac, ${ }^{62, \downarrow}$ G. Volpi ${ }^{17, \dagger}$ P. Wagner, ${ }^{40, \dagger}$ H. D. Wahl, ${ }^{97, \sharp}$ R. Wallny, ${ }^{15, g, \dagger}$ M. H. L. S. Wang, ${ }^{15, \sharp}$ S. M. Wang, ${ }^{1, \dagger}$ J. Warchol, ${ }^{103, \ddagger}$ D. Waters ${ }^{28, \dagger}$ G. Watts, ${ }^{123, \ddagger}$ M. Wayne, ${ }^{103, \ddagger}$ J. Weichert ${ }^{76, \ddagger}$ L. Welty-Rieger, ${ }^{100,}$ W. C. Wester III,,${ }^{15, \dagger}$ D. Whiteson, ${ }^{40, u u, \dagger}$ A. B. Wicklund, ${ }^{2, \dagger}$ S. Wilbur, ${ }^{7, \dagger}$ H. H. Williams, ${ }^{40, \dagger}$ M. R. J. Williams, ${ }^{101, v v, \downarrow}$ 
G. W. Wilson, ${ }^{105, \$}$ J. S. Wilson, ${ }^{31, \dagger}$ P. Wilson, ${ }^{15, \dagger}$ B. L. Winer, ${ }^{35, \dagger}$ P. Wittich, ${ }^{15, u, \dagger}$ M. Wobisch, ${ }^{106, \$}$ S. Wolbers, ${ }^{15, \uparrow}$ H. Wolfe, ${ }^{35, \dagger}$ D. R. Wood,${ }^{107, \star}$ T. Wright,${ }^{31, \dagger}$ X. Wu,${ }^{18, \dagger}$ Z. Wu,${ }^{5, \dagger}$ T. R. Wyatt, ${ }^{94,}$ Y. Xie, ${ }^{15, \$}$ R. Yamada, ${ }^{15, \downarrow}$ K. Yamamoto, ${ }^{37, \dagger}$ D. Yamato, ${ }^{37, \dagger}$ S. Yang,${ }^{59,}$ T. Yang,,${ }^{15,}$ U. K. Yang, ${ }^{25, \dagger}$ Y. C. Yang, ${ }^{25, \dagger}$ W.-M. Yao,${ }^{26, \dagger}$ T. Yasuda, ${ }^{15, \$}$ Y. A. Yatsunenko, ${ }^{13, \sharp}$

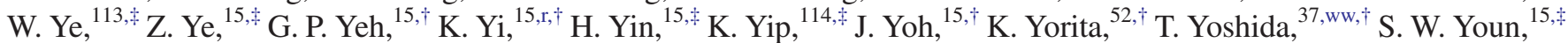
G. B. Yu, ${ }^{14, \dagger}$ I. Yu, ${ }^{25, \dagger}$ J. M. Yu, ${ }^{31, \$}$ A. M. Zanetti, ${ }^{48 a, \dagger}$ Y. Zeng, ${ }^{14, \dagger}$ J. Zennamo, ${ }^{11,+\hbar}$ T. G. Zhao, ${ }^{94, \hbar}$ B. Zhou, ${ }^{31, \$}$ C. Zhou, ${ }^{14, \dagger}$ J. Zhu, ${ }^{31, \$}$ M. Zielinski, ${ }^{44, \$}$ D. Zieminska, ${ }^{101, \$}$ L. Zivkovic, ${ }^{69, \$}$ and S. Zucchelli ${ }^{6 a, 6 b, \dagger}$

$(\mathrm{CDF} \text { Collaboration })^{\dagger}$

(D0 Collaboration) $)^{\ddagger}$

${ }^{1}$ Institute of Physics, Academia Sinica, Taipei, Taiwan 11529, Republic of China

${ }^{2}$ Argonne National Laboratory, Argonne, Illinois 60439, USA

${ }^{3}$ University of Athens, 15771 Athens, Greece

${ }^{4}$ Institut de Fisica d'Altes Energies, ICREA, Universitat Autonoma de Barcelona, E-08193, Bellaterra (Barcelona), Spain

${ }^{5}$ Baylor University, Waco, Texas 76798, USA

${ }^{6 a}$ Istituto Nazionale di Fisica Nucleare Bologna

${ }^{6 \mathrm{~b}}$ University of Bologna, I-40127 Bologna, Italy

${ }^{7}$ University of California Davis, Davis, California 95616, USA

${ }^{8}$ University of California Los Angeles, Los Angeles, California 90024, USA

${ }^{9}$ Instituto de Fisica de Cantabria, CSIC-University of Cantabria, 39005 Santander, Spain

${ }^{10}$ Carnegie Mellon University, Pittsburgh, Pennsylvania 15213, USA

${ }^{11}$ Enrico Fermi Institute, University of Chicago, Chicago, Illinois 60637, USA

${ }^{12}$ Comenius University, 84248 Bratislava, Slovakia; Institute of Experimental Physics, 04001 Kosice, Slovakia

${ }^{13}$ Joint Institute for Nuclear Research, RU-141980 Dubna, Russia

${ }^{14}$ Duke University, Durham, North Carolina 27708, USA

${ }^{15}$ Fermi National Accelerator Laboratory, Batavia, Illinois 60510, USA

${ }^{16}$ University of Florida, Gainesville, Florida 32611, USA

${ }^{17}$ Laboratori Nazionali di Frascati, Istituto Nazionale di Fisica Nucleare, I-00044 Frascati, Italy

${ }^{18}$ University of Geneva, $\mathrm{CH}-1211$ Geneva 4, Switzerland

${ }^{19}$ Glasgow University, Glasgow G12 8QQ, United Kingdom

${ }^{20}$ Harvard University, Cambridge, Massachusetts 02138, USA

${ }^{21}$ Division of High Energy Physics, Department of Physics, University of Helsinki, FIN-00014, Helsinki, Finland;

Helsinki Institute of Physics, FIN-00014, Helsinki, Finland

${ }^{22}$ University of Illinois, Urbana, Illinois 61801, USA

${ }^{23}$ The Johns Hopkins University, Baltimore, Maryland 21218, USA

${ }^{24}$ Institut für Experimentelle Kernphysik, Karlsruhe Institute of Technology, D-76131 Karlsruhe, Germany

${ }^{25}$ Center for High Energy Physics: Kyungpook National University, Daegu 702-701, Korea;

Seoul National University, Seoul 151-742, Korea; Sungkyunkwan University, Suwon 440-746, Korea;

Korea Institute of Science and Technology Information, Daejeon 305-806, Korea;

Chonnam National University, Gwangju 500-757, Korea; Chonbuk National University, Jeonju 561-756, Korea; Ewha Womans University, Seoul, 120-750, Korea

${ }^{26}$ Ernest Orlando Lawrence Berkeley National Laboratory, Berkeley, California 94720, USA

${ }^{27}$ University of Liverpool, Liverpool L69 7ZE, United Kingdom

${ }^{28}$ University College London, London WC1E 6BT, United Kingdom

${ }^{29}$ Centro de Investigaciones Energeticas Medioambientales y Tecnologicas, E-28040 Madrid, Spain

${ }^{30}$ Massachusetts Institute of Technology, Cambridge, Massachusetts 02139, USA

${ }^{31}$ University of Michigan, Ann Arbor, Michigan 48109, USA

${ }^{32}$ Michigan State University, East Lansing, Michigan 48824, USA

${ }^{33}$ Institution for Theoretical and Experimental Physics, ITEP, Moscow 117259, Russia

${ }^{34}$ University of New Mexico, Albuquerque, New Mexico 87131, USA

${ }^{35}$ The Ohio State University, Columbus, Ohio 43210, USA

${ }^{36}$ Okayama University, Okayama 700-8530, Japan

${ }^{37}$ Osaka City University, Osaka 558-8585, Japan

${ }^{38}$ University of Oxford, Oxford OX1 3RH, United Kingdom

${ }^{39 a}$ Istituto Nazionale di Fisica Nucleare, Sezione di Padova

${ }^{39 b}$ University of Padova, I-35131 Padova, Italy

${ }^{40}$ University of Pennsylvania, Philadelphia, Pennsylvania 19104, USA

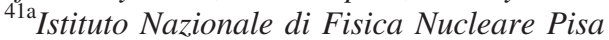




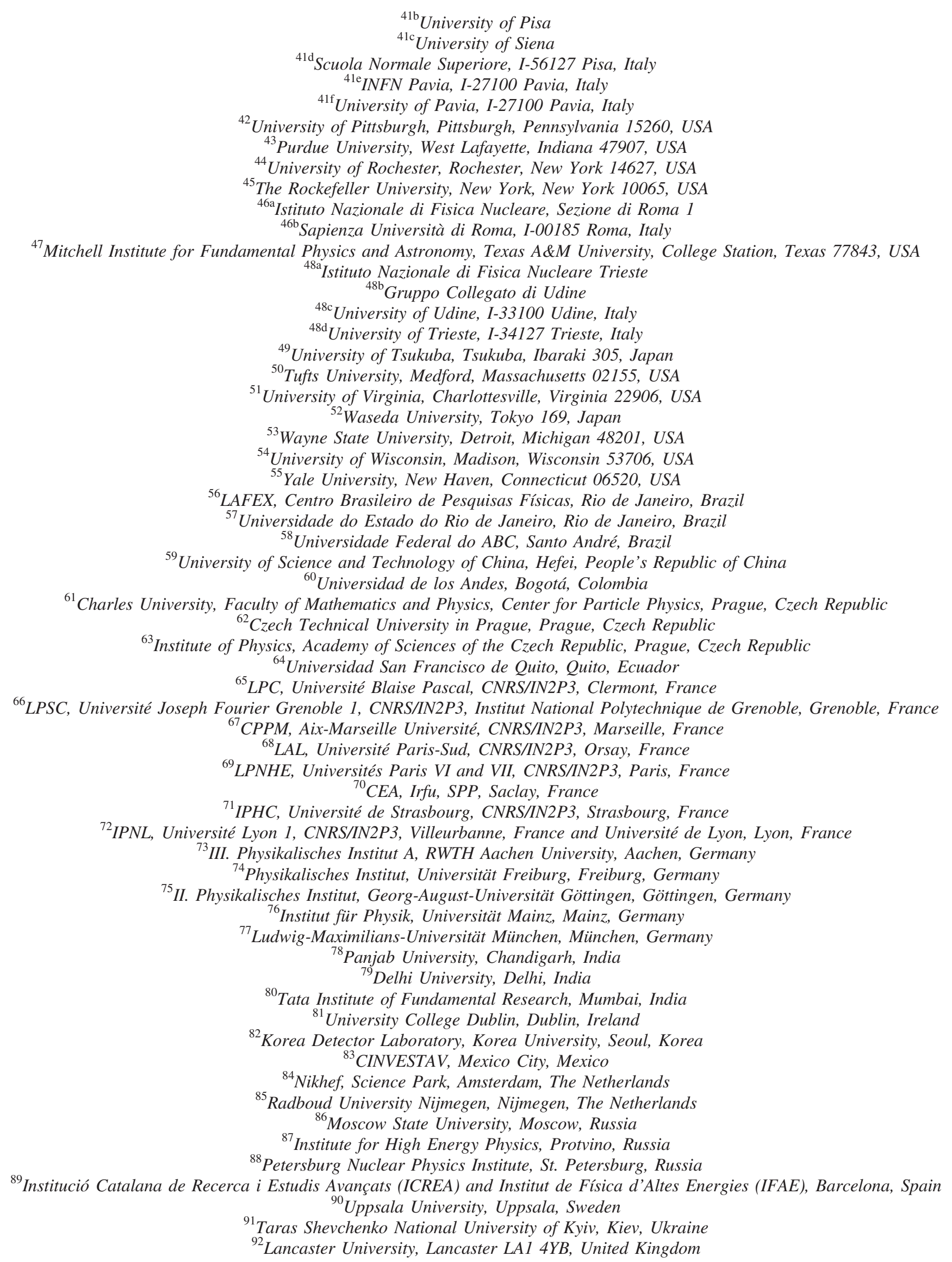




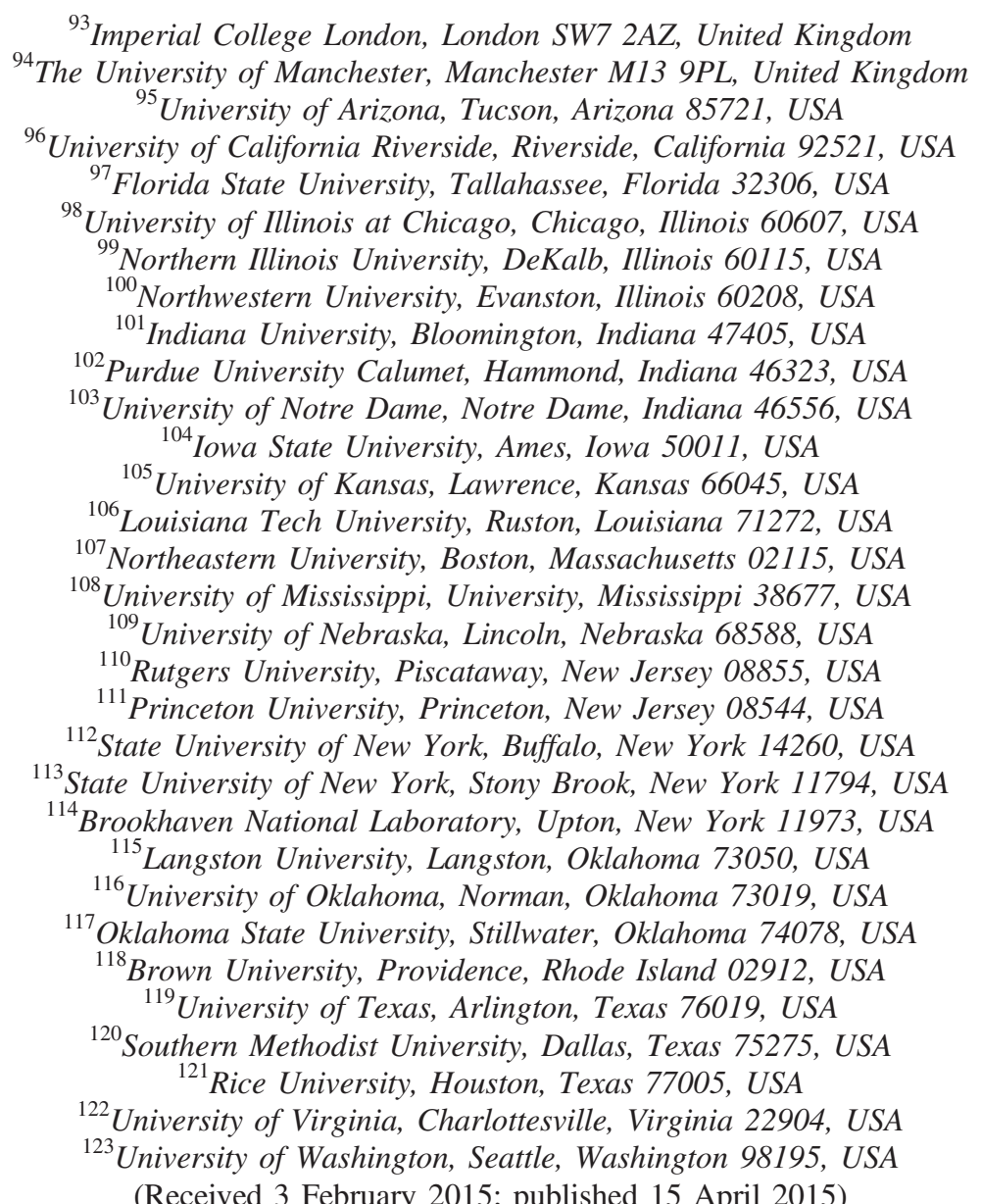

Combined constraints from the CDF and D0 Collaborations on models of the Higgs boson with exotic spin $J$ and parity $P$ are presented and compared with results obtained assuming the standard model value $J^{P}=0^{+}$. Both collaborations analyzed approximately $10 \mathrm{fb}^{-1}$ of proton-antiproton collisions with a center-of-mass energy of $1.96 \mathrm{TeV}$ collected at the Fermilab Tevatron. Two models predicting exotic Higgs bosons with $J^{P}=0^{-}$and $J^{P}=2^{+}$are tested. The kinematic properties of exotic Higgs boson production in association with a vector boson differ from those predicted for the standard model Higgs boson. Upper limits at the $95 \%$ credibility level on the production rates of the exotic Higgs bosons, expressed as fractions of the standard model Higgs boson production rate, are set at 0.36 for both the $J^{P}=0^{-}$hypothesis and the $J^{P}=2^{+}$hypothesis. If the production rate times the branching ratio to a bottom-antibottom pair is the same as that predicted for the standard model Higgs boson, then the exotic bosons are excluded with significances of 5.0 standard deviations and 4.9 standard deviations for the $J^{P}=0^{-}$and $J^{P}=2^{+}$ hypotheses, respectively.

DOI: $10.1103 /$ PhysRevLett.114.151802

PACS numbers: 14.80.Ec, 13.85.Rm, 14.80.Bn

The Higgs boson discovered by the ATLAS [1] and CMS [2] Collaborations in 2012 using data produced in proton-proton collisions at the Large Hadron Collider (LHC) at CERN allows many stringent tests of the

Published by the American Physical Society under the terms of the Creative Commons Attribution 3.0 License. Further distribution of this work must maintain attribution to the author(s) and the published article's title, journal citation, and DOI. electroweak symmetry breaking in the standard model (SM) and extensions to the SM to be performed. To date, measurements of the Higgs boson's mass and width [3-6], its couplings to other particles [3,7-11], and its spin and parity quantum numbers $J$ and $P$ [10-16] are consistent with the expectations for the SM Higgs boson. The CDF and D0 Collaborations at the Fermilab Tevatron observed a 3.0 standard deviation (s.d.) excess of events consistent with a Higgs boson signal, largely driven by those channels sensitive to the decay of the Higgs boson to bottom quarks 
$(H \rightarrow b \bar{b})[17,18]$. The Tevatron data are also consistent with the predictions for the properties of the SM Higgs boson [18-22].

The authors of Ref. [23] proposed to use the Tevatron data to test models for the Higgs boson with exotic spin and parity, using events in which the exotic Higgs boson $X$ is produced in association with a $W$ or a $Z$ boson and decays to a bottom-antibottom quark pair $X \rightarrow b \bar{b}$. This proposal used two of the spin and parity models in Ref. [24], one with a pseudoscalar $J^{P}=0^{-}$state and the other with a gravitonlike $J^{P}=2^{+}$state. For the SM Higgs boson, which has $J^{P}=0^{+}$, the differential production rate near threshold is linear in $\beta$, where $\beta=2 p / \sqrt{\hat{s}}, p$ is the momentum of the $X$ boson in the $V X(V=W$ or $Z)$ reference frame, and $\sqrt{\hat{s}}$ is the total energy of the $V X$ system in its rest frame. For the pseudoscalar model, the dependence is proportional to $\beta^{3}$. For the gravitonlike model, the dependence is proportional to $\beta^{5}$; however, not all $J^{P}=2^{+}$models share this $\beta^{5}$ factor [23]. These powers of $\beta$ alter the kinematic distributions of the observable decay products of the vector boson and the Higgs-like boson $X$, most notably the invariant mass of the $V X$ system, which has a higher average value in the $J^{P}=0^{-}$hypothesis than in the $\mathrm{SM} 0^{+}$case and higher still in the $J^{P}=2^{+}$hypothesis. These models predict neither the production rates nor the decay branching fractions of the $X$ particles.

The ATLAS and CMS Collaborations recently reported strong evidence for Higgs boson decays to fermions [25-30], with sensitivity dominated by the $H \rightarrow \tau^{+} \tau^{-}$ decay mode, though they have not yet performed spin and parity tests using fermionic decays. The particle decaying fermionically for which the Tevatron also found evidence might not be the same as the particle discovered through its bosonic decays at the LHC. Tests of the spin and parity [23] with Tevatron data therefore provide unique information on the identity and properties of the new particle or particles. The CDF and D0 Collaborations have reoptimized their SM Higgs boson searches to test the exotic Higgs boson models in the $W H \rightarrow \ell \nu b \bar{b}[31,32]$, $\mathrm{ZH} \rightarrow \ell^{+} \ell^{-} b \bar{b}[33,34]$, and $W H+Z H \rightarrow E_{T} b \bar{b}[35,36]$ channels, where $\ell=e$ or $\mu$ and $E_{T}$ is the missing transverse energy [37]. In this Letter, we report a combination of the CDF [21] and D0 [22] studies of the $J^{P}$ assignments of the state $X$, with mass $m_{X}=125 \mathrm{GeV} / c^{2}$, in the $X \rightarrow b \bar{b}$ decay.

The CDF and D0 detectors are multipurpose solenoidal spectrometers surrounded by hermetic calorimeters and muon detectors designed to study the products of $1.96 \mathrm{TeV}$ proton-antiproton $(p \bar{p})$ collisions [38,39]. All searches combined here use the complete Tevatron data sample, which, after data quality requirements, corresponds to 9.45-9.7 $\mathrm{fb}^{-1}$ of integrated luminosity, depending on the experiment and the search channel.

Standard model Higgs boson signal events are simulated using the leading-order calculation from PYTHIA [40], with
CTEQ5L (CDF) and CTEQ6L1 (D0) [41] parton distribution functions (PDFs). The $J^{P}=0^{-}$and $2^{+}$signal samples are generated using MADGRAPH 5 version 1.4.8.4 [42], with modifications provided by the authors of Ref. [23]. Subsequent particle showering is modeled by PYTHIA. We normalize the SM Higgs boson rate predictions to the highest-order calculations available. The $\mathrm{WH}$ and $\mathrm{ZH}$ cross sections are calculated at next-to-next-to-leadingorder (NNLO) precision in the strong interaction and next-to-leading-order (NLO) precision in the electroweak corrections [43-46]. We use the branching fractions for Higgs boson decay from Ref. [47]. These rely on calculations using HDECAY [48] and PROPHECY4F [49].

The predictions of the dominant background rates and kinematic distributions are treated in the following way. Diboson ( $W W, W Z$, and $Z Z$ ) Monte Carlo (MC) samples are normalized using the NLO calculations from MCFM [50]. For $t \bar{t}$, we use a production cross section of $7.04 \pm$ $0.70 \mathrm{pb}$ [51], which is based on a top quark mass of $173 \mathrm{GeV} / c^{2}$ [52] and MSTW 2008 NNLO PDFs [53]. The single top quark production cross section is assumed to be $3.15 \pm 0.31 \mathrm{pb}$ [54]. For details of the generators used, see Ref. [55]. Data-driven methods are used to normalize the $V$ plus light-flavor and heavy-flavor jet backgrounds [60] using $V$ data events containing no $b$-tagged jets [61], which have negligible signal content $[62,63]$. The MC modeling of the kinematic distributions of the background predictions is described in Refs. [31-36].

The event selections are similar (CDF), or identical (D0), to those used in their SM counterparts [31-36]. For the $W H \rightarrow \ell \nu b \bar{b}$ analyses, events are selected with one identified lepton $\left(e\right.$ or $\mu$ ), jets, and large $E_{T}$. For the CDF $W H \rightarrow \ell \nu b \bar{b}$ analysis, only events with two jets are used. Events are classified into separate categories based on the quality of the identified lepton. Separate categories are used for events with a high-quality muon or central electron candidate, an isolated track, or a forward electron candidate. Within the lepton categories, five exclusive $b$-tag categories, comprising two single-tag and three double-tag categories, are formed. The multivariate $b$ tagger used by CDF [64] was trained on SM Higgs boson signal MC events. Few of these events contained jets with transverse energy $E_{T}>200 \mathrm{GeV}$, and thus, the tagger does not perform well for such jets. Hence, only jets with $E_{T}<$ $200 \mathrm{GeV}$ are considered. For the D0 $W H \rightarrow \ell \nu b \bar{b}$ analysis, events are selected with two or three jets. The data are split by lepton flavor and jet multiplicity (two or three jet subchannels) and by the output of the $b$-tagging algorithm applied to all selected jets in the event. This channel, along with the other two D0 channels, uses a multivariate $b$-tagging algorithm $[65,66]$. Four exclusive $b$-tag categories, one single-tag and three double-tag, are formed. In the SM Higgs boson search, boosted decision trees are used as the final discriminating variables; here, they are used to 
further subdivide the selected data sample into high- and low-purity categories.

The $Z H \rightarrow \ell^{+} \ell^{-} b \bar{b}$ analyses require two isolated leptons and at least two jets. The CDF analysis separates events into one single- and three double- $b$-tag samples and uses neural networks to select loose dielectron and dimuon candidates. The jet energies are corrected for $\mathbb{E}_{T}$ using a neural network [67]. The CDF analysis uses a multistep discriminant based on neural networks, where two discriminant functions are used to define three separate regions of the final discriminant function. The D0 $\mathrm{ZH} \rightarrow$ $\ell^{+} \ell^{-} b \bar{b}$ analysis separates events into nonoverlapping samples of events with either a single or double $b$ tag. To increase the signal acceptance, the selection criteria for one of the leptons are loosened to include isolated tracks not reconstructed in the muon detector and electron candidates from the intercryostat region of the D0 detector. Combined with the dielectron and dimuon categories, these provide four independent lepton subchannels. A kinematic fit is used to optimize reconstruction. Random forests (RF) of decision trees $[68,69]$ are used to provide the final variables in the SM Higgs boson search. The first RF is designed to discriminate against $t \bar{t}$ events and divides events into $t \bar{t}$-enriched and $t \bar{t}$-depleted single-tag and double-tag regions. Only events in the $t \bar{t}$-depleted regions are considered in this study. These regions contain approximately $94 \%$ of the SM signal.

For the $Z H \rightarrow \nu \bar{\nu} b \bar{b}$ analyses, the selections used by $\mathrm{CDF}$ and D0 are similar to the $W H$ selections, except that all events with isolated leptons are rejected and more stringent techniques are applied to reject the multijet background. In a sizable fraction of $W H \rightarrow \ell \nu b \bar{b}$ signal events, the lepton is undetected. Such events often are selected in the $Z H \rightarrow \nu \bar{\nu} b \bar{b}$ samples, so these analyses are also referred to as $V H \rightarrow E_{T} b \bar{b}$. The CDF analysis uses three nonoverlapping $b$-tag categories (two double- and one single-tag) and two jet categories (two- or three-jet events), giving a total of six subchannels. In the D0 analysis, exactly two jets are required and two exclusive double-tag categories are defined using the sum of the $b$-tagging outputs for each of the two selected jets.

Both CDF and D0 have a 50\% larger acceptance for the $J^{P}=0^{-}$and $2^{+}$signals in the $Z H \rightarrow \nu \bar{\nu} b \bar{b}$ analyses compared with the SM Higgs boson signal, largely due to the fact that the exotic signal events are more likely to pass the trigger thresholds for $E_{T}$, a consequence of the larger average $V X$ invariant masses. The other two channels, $W H \rightarrow \ell \nu b \bar{b}$ and $Z H \rightarrow \ell^{+} \ell^{-} b \bar{b}$, do not benefit as much from the additional $E_{T}$ in these events, as they rely on the lepton triggers, which are more efficient than the $E_{T}$ triggers in the relevant kinematic regions.

Unlike their SM counterparts, these analyses are optimized to distinguish the $J^{P}=0^{-}$and the $J^{P}=2^{+}$hypotheses from the $\mathrm{SM} 0^{+}$hypothesis. The exotic particles are considered either in addition to, or replacing, the SM Higgs boson. A mixture of all three states is not considered.

The CDF multivariate analysis (MVA) discriminants were newly trained to separate the exotic Higgs boson signals from the SM backgrounds [21]. In the $W H \rightarrow \ell \nu b \bar{b}$ and $V H \rightarrow E_{T} b \bar{b}$ channels, events classified as backgroundlike by the new discriminants are then classified according to the SM-optimized MVA discriminants in order to improve the performance of tests between the SM and exotic hypotheses.

Depending on the channel, D0 uses either the reconstructed dijet mass or the MVA used in the SM Higgs boson search to separate events into high- and low-purity samples. The mass of the $V X$ system is then used to discriminate between the exotic and SM hypotheses [22]. For the $\mathrm{ZH} \rightarrow$ $\ell \ell b \bar{b}$ analysis, the invariant mass of the two leptons and the two highest $p_{T}$ jets is used. For the $\ell \nu b \bar{b}$ and $\nu \nu b \bar{b}$ final states, the transverse mass $M_{T}$ is used, where $M_{T}^{2}=\left(E_{T}^{V}+\right.$ $\left.E_{T}^{X}\right)^{2}-\left(\vec{p}_{T}^{V}+\vec{p}_{T}^{X}\right)^{2}$ and the transverse momenta of the $Z$ and $W$ bosons are taken to be $\vec{p}_{T}^{Z}=\vec{E}_{T}$ and $\vec{p}_{T}^{W}=\vec{E}_{T}+\vec{p}_{T}^{\ell}$, respectively.

The number of contributing channels is large, and their sensitivities vary from one to another. To visualize the data in a way that emphasizes the sensitivity to the exotic signals, we follow Ref. [18]. Bins of the final discriminant for all channels are ordered by increasing signal-to-background ratio $(s / b)$ and are shown in comparison with predicted yields from signal and background processes for the $J^{P}=0^{-}$and $2^{+}$searches in Fig. 1 separately. The backgrounds are fit to the data in each case, allowing the systematic uncertainties to vary within their a priori constraints. The exotic signals are normalized to the SM cross section times branching ratio multiplied by an exotic-signal scaling factor $\mu_{\text {exotic }}$. They are shown in Fig. 1 with $\mu_{\text {exotic }}=1$. The scaling factor for the SM Higgs boson signal is denoted by $\mu_{\mathrm{SM}}$. A value of 1 for either $\mu_{\mathrm{SM}}$ or $\mu_{\text {exotic }}$ corresponds to a cross section times branching ratio as predicted for the SM Higgs boson. Both figures show agreement between the background predictions and the observed data over 5 orders of magnitude with no evidence for an excess of exotic signal-like candidates.

We follow Ref. [18] and perform both Bayesian and modified frequentist calculations of the upper limits on exotic $X$ boson production with and without SM Higgs production, best-fit cross sections allowing for the simultaneous presence of a SM Higgs boson and an exotic $X$ boson, and hypothesis tests for signals assuming various production rate times branching ratio values for the exotic bosons. Both methods use likelihood calculations based on Poisson probabilities that include SM background processes and signal predictions for the SM Higgs and exotic bosons multiplied by their respective scaling factors $\mu_{\mathrm{SM}}$ and $\mu_{\text {exotic. }}$ Systematic uncertainties on the predicted rates and on the shapes of the distributions and their correlations are treated as described in Ref. [18]. Theoretical uncertainties in cross 

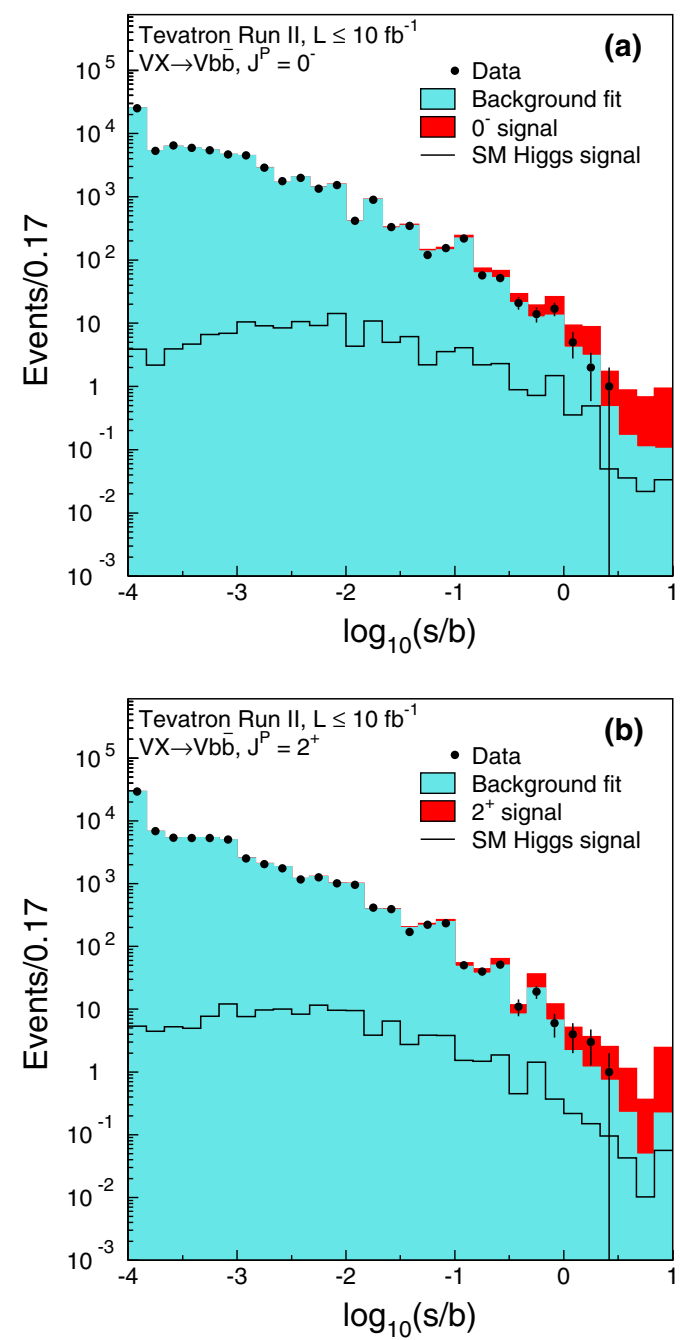

FIG. 1 (color online). Distribution of $\log _{10}(s / b)$ for CDF and D0 data from all contributing search channels, for (a) the $J^{P}=0^{-}$ search and (b) the $J^{P}=2^{+}$search. The data are shown with points, and the expected exotic signals are shown with $\mu_{\text {exotic }}=1$ stacked on top of the fitted backgrounds. The solid lines denote the predictions for the SM Higgs boson and are not stacked. Underflows and overflows are collected into the leftmost and rightmost bins, respectively.

sections and branching ratios are considered fully correlated between CDF and D0 and between analysis samples. The uncertainties on the measurements of the integrated luminosities, which are used to normalize the expected signal yields and the MC-based background rates, are $6.0 \%(\mathrm{CDF})$ and $6.1 \%(\mathrm{D} 0)$. Of these values, $4 \%$ arises from the inelastic $p \bar{p}$ cross section [70], which is fully correlated between CDF and D0. The dominant uncertainties on the backgrounds are constrained by the data in low $s / b$ regions of the discriminant distributions. Different methods were used by CDF and D0 to estimate $V+$ jets and multijet backgrounds, and so their uncertainties are considered uncorrelated. Similarly, the uncertainties on the data-driven estimates of the $b$-tag efficiencies are considered uncorrelated between CDF and D0, as are the uncertainties on the jet energy scales, the trigger efficiencies, and lepton identification efficiencies. We quote Bayesian upper limits and best-fit cross sections assuming uniform priors for non-negative signal cross sections, and we use the modified frequentist method to perform the hypothesis tests. Systematic uncertainties are parametrized by nuisance parameters with Gaussian priors, truncated so that no predicted yield for any process in any search channel is negative.

For both the $J^{P}=0^{-}$and $2^{+}$models, we compute two 95\% credibility upper limits on $\mu_{\text {exotic }}$, one assuming $\mu_{\mathrm{SM}}=1$ and the other assuming $\mu_{\mathrm{SM}}=0$. The expected limits are the median expectations, assuming no exotic boson is present. The results are listed in Table I. Twodimensional credibility regions, which are the smallest regions containing $68 \%$ and $95 \%$ of the posterior probabilities, are shown in Fig. 2 . The points in the $\left(\mu_{\mathrm{SM}}, \mu_{\text {exotic }}\right)$ planes that maximize the posterior probability densities are shown as the best-fit values. These best-fit values are $\left(\mu_{\mathrm{SM}}=1.0, \mu_{0^{-}}=0\right)$ for the search for the $J^{P}=0^{-}$state and $\left(\mu_{\mathrm{SM}}=1.1, \mu_{2^{+}}=0\right)$ for the search for the $J^{P}=2^{+}$ state. We also derive upper limits on the fraction $f_{J P}=\mu_{\text {exotic }} /\left(\mu_{\text {exotic }}+\mu_{\mathrm{SM}}\right)$, as functions of the total $\mu=\mu_{\text {exotic }}+\mu_{\mathrm{SM}}$, assuming a uniform prior probability density in non-negative $f_{J P}$, extended to include fractions larger than 1.0 in order not to saturate the limits at $f_{J P}=0.95$ for $\mu<0.6$, where the test is weak. The results are shown in Fig. 3.

In the modified frequentist approach $[71,72]$ we compute $p$ values for the discrete two-hypothesis tests, the SM Higgs boson hypothesis (the "null" hypothesis) $\left(\mu_{\mathrm{SM}}=1\right.$, $\left.\mu_{\text {exotic }}=0\right)$ and the exotic ("test") hypothesis $\left(\mu_{\mathrm{SM}}=0\right.$, $\mu_{\text {exotic }}=1$ ), both assuming that SM background processes are present. The choice of setting $\mu_{\text {exotic }}=1$ in the test hypothesis is arbitrary; the sensitivity of the test is reduced if a smaller value is assumed. We use the log-likelihood ratio, LLR, defined to be $-2 \ln [p$ (data|test) $/ p$ (data|null) $]$, where the numerator and denominator are maximized over systematic uncertainty variations [18]. The LLR distributions are shown in the Supplemental Material [73]. We define the $p$ values $p_{\text {null }}=P\left(\operatorname{LLR} \leq \operatorname{LLR}_{\text {obs }} \mid \mathrm{SM}\right)$

TABLE I. Observed and median expected Bayesian upper limits at the $95 \%$ credibility level on $\mu_{\text {exotic }}$ for the pseudoscalar $\left(J^{P}=0^{-}\right)$and gravitonlike $\left(J^{P}=2^{+}\right)$boson models, assuming either that the SM Higgs boson is also present $\left(\mu_{\mathrm{SM}}=1\right)$ or absent $\left(\mu_{\mathrm{SM}}=0\right)$.

\begin{tabular}{lcc}
\hline \hline Channel & $\begin{array}{c}\text { Observed } \\
\left(\text { limit } / \sigma_{\mathrm{SM}}\right)\end{array}$ & $\begin{array}{c}\text { Median expected } \\
\left(\text { limit } / \sigma_{\mathrm{SM}}\right)\end{array}$ \\
\hline$J^{P}=0^{-}, \mu_{\mathrm{SM}}=0$ & 0.36 & 0.32 \\
$J^{P}=0^{-}, \mu_{\mathrm{SM}}=1$ & 0.29 & 0.32 \\
$J^{P}=2^{+}, \mu_{\mathrm{SM}}=0$ & 0.36 & 0.33 \\
$J^{P}=2^{+}, \mu_{\mathrm{SM}}=1$ & 0.31 & 0.34 \\
\hline \hline
\end{tabular}



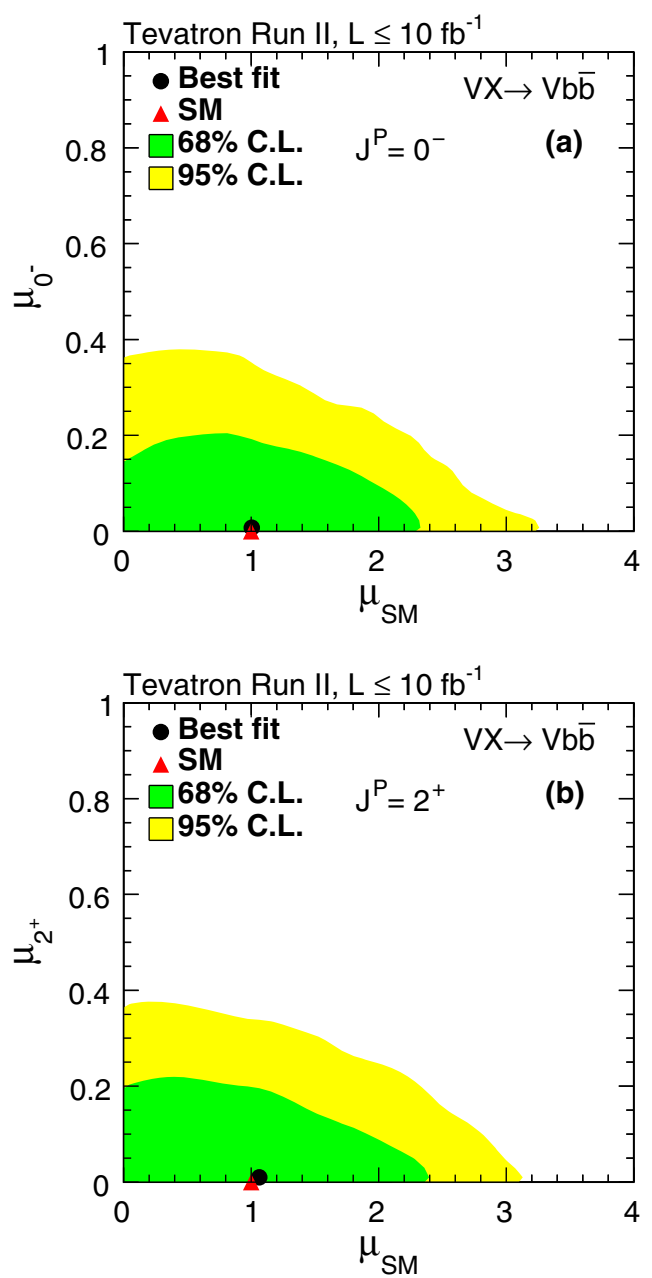

FIG. 2 (color online). Two-dimensional credibility regions in the $\left(\mu_{\text {exotic }}, \mu_{\mathrm{SM}}\right)$ plane, for the combined CDF and D0 searches for (a) the pseudoscalar $\left(J^{P}=0^{-}\right)$boson and (b) the gravitonlike $\left(J^{P}=2^{+}\right)$boson

and $p_{\text {test }}=P\left(\operatorname{LLR} \geq \mathrm{LLR}_{\text {obs }} \mid\right.$ exotic $)$. The median expected $p$ values $p_{\text {null,med }}^{\text {exotic }}$ in the test hypothesis and $p_{\text {test,med }}^{\mathrm{SM}}$ in the SM hypothesis quantify the sensitivities of the twohypothesis tests for exclusion and discovery, respectively. Table II lists these $p$ values for both exotic models as well as $\mathrm{CL}_{\mathrm{s}}=p_{\text {test }} /\left(1-p_{\text {null }}\right)$ [71] for the Tevatron combination. To compute $p_{\text {test }}$ and the expected values of $p_{\text {null }}$ and $p_{\text {test }}$, Wilks's theorem is used [74].

The similarity of the limits and $p$ values obtained for the $J^{P}=0^{-}$and the $J^{P}=2^{+}$searches is expected since the exotic models predict excesses in similar portions of kinematic space.

In summary, we combine CDF's and D0's tests for the presence of a pseudoscalar Higgs boson with $J^{P}=0^{-}$and a gravitonlike boson with $J^{P}=2^{+}$in the $W X \rightarrow \ell \nu b \bar{b}$, $Z X \rightarrow \ell^{+} \ell^{-} b \bar{b}$, and $V X \rightarrow E_{T} b \bar{b}$ search channels using models described in Ref. [23]. The masses of the exotic bosons are assumed to be $125 \mathrm{GeV} / c^{2}$. No evidence is seen for either exotic particle, either in place of the

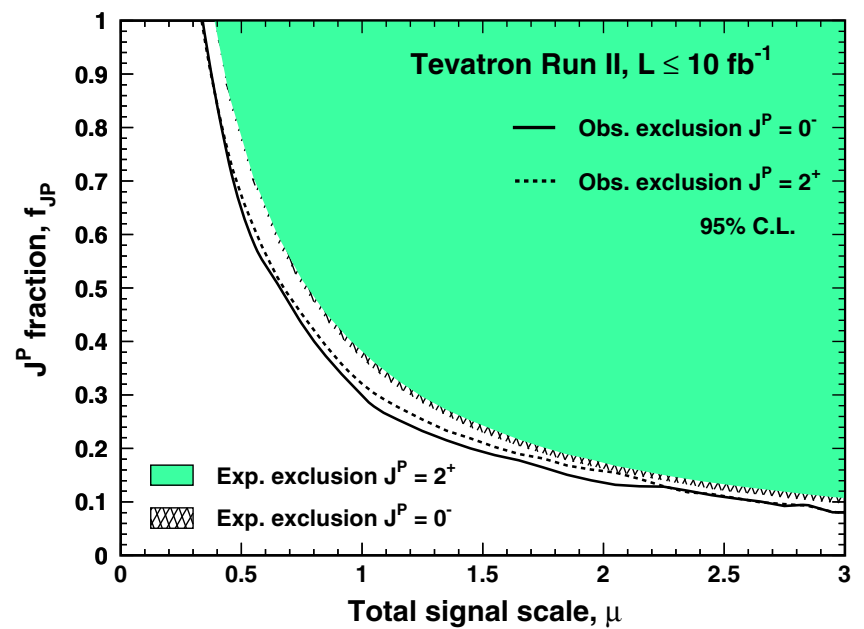

FIG. 3 (color online). Observed and expected upper limits at the $95 \%$ C.L. on the fraction of exotic boson production for the $J^{P}=0^{-}$and $2^{+}$hypotheses.

SM Higgs boson or produced in a mixture with a $J^{P}=0^{+}$Higgs boson. In both searches, the best-fit cross section times the decay branching ratio into a bottomantibottom quark pair of a $J^{P}=0^{+}$signal component is consistent with the prediction of the SM Higgs boson. The Bayesian posterior probability densities for the $J^{P}=0^{-}$and $J^{P}=2^{+}$searches are shown in the Supplemental Material [73].

Upper limits at $95 \%$ credibility on the rate of the production of an exotic Higgs boson in the absence of a SM $J^{P}=0^{+}$signal are set at 0.36 times the SM Higgs production rate for both the $J^{P}=0^{-}$and the $J^{P}=2^{+}$ hypotheses. If the production rate of the hypothetical exotic

TABLE II. Observed (obs) and median expected (med) LLR values and $p$ values for the combined CDF and D0 searches for the pseudoscalar $\left(J^{P}=0^{-}\right)$boson and the graviton-like $\left(J^{P}=2^{+}\right)$boson. The $p$ values are listed, and the corresponding significances in units of standard deviations, using a one-sided Gaussian tail calculation, are given in parentheses. The two hypotheses tested are $\left(\mu_{\mathrm{SM}}, \mu_{\text {exotic }}\right)=(1,0)$ and $(0,1)$ for the SM and the exotic models, respectively.

\begin{tabular}{lcc}
\hline \hline Analysis & $J^{P}=0^{-}$ & $J^{P}=2^{+}$ \\
\hline $\mathrm{LLR}_{\text {obs }}$ & 27.1 & 25.7 \\
$\mathrm{LLR}_{\text {med }}^{\mathrm{SM}}$ & 23.7 & 21.8 \\
$\mathrm{LLR}_{\text {med }}^{\text {exotic }}$ & -29.9 & -29.6 \\
$p_{\text {null }}$ & $0.63(-0.34)$ & $0.66(-0.41)$ \\
$p_{\text {null,med }}^{\text {exotic }}$ & $1.8 \times 10^{-8}(5.5)$ & $1.9 \times 10^{-8}(5.5)$ \\
$p_{\text {test }}$ & $9.4 \times 10^{-8}(5.2)$ & $1.9 \times 10^{-7}(5.1)$ \\
$p_{\text {test, med }}^{\text {SM }}$ & $4.7 \times 10^{-7}(4.9)$ & $1.2 \times 10^{-6}(4.7)$ \\
$\mathrm{CL}_{\mathrm{s}}$ & $2.6 \times 10^{-7}(5.0)$ & $5.6 \times 10^{-7}(4.9)$ \\
$\mathrm{CL}_{\mathrm{s}, \text { med }}^{\text {SM }}$ & $9.4 \times 10^{-7}(4.8)$ & $2.3 \times 10^{-6}(4.6)$ \\
\hline \hline
\end{tabular}


particle times its branching ratio to a bottom-antibottom quark pair is the same as that predicted for the SM Higgs boson, then the exotic models are excluded with significances of 5.0 s.d. and 4.9 s.d. for the $J^{P}=0^{-}$and $2^{+}$ hypotheses, respectively.

We thank the Fermilab staff and technical staffs of the participating institutions for their vital contributions. We acknowledge support from the Department of Energy and the National Science Foundation (United States of America), the Australian Research Council (Australia), the National Council for the Development of Science and Technology and the Carlos Chagas Filho Foundation for the Support of Research in the State of Rio de Janeiro (Brazil), the Natural Sciences and Engineering Research Council (Canada), the China Academy of Sciences, the National Natural Science Foundation of China, and the National Science Council of the Republic of China (China), the Administrative Department of Science, Technology and Innovation (Colombia), the Ministry of Education, Youth and Sports (Czech Republic), the Academy of Finland (Finland), the Alternative Energies and Atomic Energy Commission and the National Center for Scientific Research/National Institute of Nuclear and Particle Physics (France), the Bundesministerium für Bildung und Forschung (Federal Ministry of Education and Research) and the Deutsche Forschungsgemeinschaft (German Research Foundation) (Germany), the Department of Atomic Energy and Department of Science and Technology (India), the Science Foundation Ireland (Ireland), the National Institute for Nuclear Physics (Italy), the Ministry of Education, Culture, Sports, Science and Technology (Japan), the Korean World Class University Program and the National Research Foundation of Korea (Korea), the National Council of Science and Technology (Mexico), the Foundation for Fundamental Research on Matter (The Netherlands), the Ministry of Education and Science of the Russian Federation, the National Research Center "Kurchatov Institute" of the Russian Federation, and the Russian Foundation for Basic Research (Russia), the Slovak R\&D Agency (Slovakia), the Ministry of Science and Innovation, and the Consolider-Ingenio 2010 Program (Spain), the Swedish Research Council (Sweden), the Swiss National Science Foundation (Switzerland), the Ministry of Education and Science of Ukraine (Ukraine), the Science and Technology Facilities Council and The Royal Society (United Kingdom), the A.P. Sloan Foundation (USA), and the European Commission Marie Curie Fellowship, Contract No. 302103.

\footnotetext{
*Deceased.

${ }^{a}$ Visitor from Augustana College, Sioux Falls, SD, USA.

${ }^{\mathrm{b}}$ Visitor from Northwestern University, Evanston, IL 60208, USA.
}

${ }^{c}$ Visitor from National Academy of Science of Ukraine (NASU), Kiev Institute for Nuclear Research (KINR).

${ }^{\mathrm{d}}$ Visitor from The University of Liverpool, Liverpool, United Kingdom.

${ }^{\mathrm{e}}$ Visitor from University of Zürich, 8006 Zürich, Switzerland.

${ }^{f}$ Visitor from Universidad Iberoamericana, Lomas de Santa Fe, C.P. 01219, Distrito Federal, México.

${ }^{\mathrm{g}}$ Visitor from ETH, 8092 Zürich, Switzerland.

${ }^{\mathrm{h}}$ Visitor from CERN, CH-1211 Geneva, Switzerland.

${ }^{\mathrm{i}}$ Visitor from Queen Mary, University of London, London, E1 4NS, United Kingdom.

${ }^{\mathrm{j}}$ Visitor from American Association for the Advancement of Science, Washington, DC 20005, USA.

${ }^{\mathrm{k}}$ Visitor from CNRS-IN2P3, Paris, F-75205, France.

${ }^{\mathrm{l}}$ Visitor from Universidad de Oviedo, E-33007 Oviedo, Spain.

${ }^{\mathrm{m}}$ Visitor from National Research Nuclear University, Moscow 115409, Russia.

${ }^{\mathrm{n}}$ Visitor from Hampton University, Hampton, VA 23668, USA.

${ }^{\circ}$ Visitor from DESY, Hamburg, Germany.

${ }^{\mathrm{p}}$ Visitor from Brookhaven National Laboratory, Upton, NY 11973, USA.

${ }^{\mathrm{q}}$ Visitor from Muons, Inc., Batavia, IL 60510, USA.

${ }^{\mathrm{r}}$ Visitor from University of Iowa, Iowa City, IA 52242, USA.

${ }^{\mathrm{s}}$ Visitor from Universidad Michoacana de San Nicolas de Hidalgo, Morelia, Mexico.

${ }^{t}$ Visitor from University College London, London, United Kingdom.

${ }^{\mathrm{u}}$ Visitor from Cornell University, Ithaca, NY 14853, USA.

${ }^{v}$ Visitor from The University of Jordan, Amman 11942, Jordan.

${ }^{\mathrm{w}}$ Visitor from Kansas State University, Manhattan, KS 66506, USA.

${ }^{\mathrm{x}}$ Visitor from University of Maryland, College Park, MD 20742, USA.

${ }^{\mathrm{y}}$ Visitor from Kinki University, Higashi-Osaka City 5778502, Japan.

${ }^{\mathrm{z}}$ Visitor from Los Alamos National Laboratory, Los Alamos, NM 87544, USA.

${ }^{a a}$ Visitor from University of Notre Dame, Notre Dame, IN 46556, USA.

${ }^{\mathrm{bb}}$ Visitor from University of Melbourne, Victoria 3010, Australia.

${ }^{\mathrm{cc}}$ Visitor from University of British Columbia, Vancouver, BC V6T 1Z1, Canada.

${ }^{\mathrm{dd}}$ Visitor from Centro de Investigacion en Computacion, IPN, Mexico City, Mexico.

${ }^{\text {ee }}$ Visitor from Institute of Physics, Academy of Sciences of the Czech Republic, 182 21, Czech Republic.

${ }^{\mathrm{ff}}$ Visitor from Istituto Nazionale di Fisica Nucleare, Sezione di Cagliari, 09042 Monserrato (Cagliari), Italy.

${ }^{\mathrm{gg}}$ Visitor from Università degli Studi di Napoli Federico I, I-80138 Napoli, Italy.

${ }^{\mathrm{hh}}$ Visitor from University College Dublin, Dublin 4, Ireland.

${ }^{\mathrm{ii}}$ Visitor from Karlsruher Institut für Technologie (KIT), Steinbuch Centre for Computing (SCC), D-76131 Karlsruhe, Germany. 
${ }^{\mathrm{jj}}$ Visitor from Massachusetts General Hospital, Boston, MA 02114, USA.

${ }^{\mathrm{kk}}$ Visitor from Harvard Medical School, Boston, MA 02114, USA.

${ }^{11}$ Visitor from SLAC, Menlo Park, CA, USA.

${ }^{\mathrm{mm}}$ Visitor from Office of Science, U.S. Department of Energy, Washington, DC 20585, USA.

${ }^{\mathrm{nn}}$ Visitor from Istituto Nazionale di Fisica Nucleare, Sezione di Lecce, Via Arnesano, I-73100 Lecce, Italy.

${ }^{00}$ Visitor from Universidad Tecnica Federico Santa Maria, 110v Valparaiso, Chile.

${ }^{\mathrm{pp}}$ Visitor from University of Cyprus, Nicosia CY-1678, Cyprus.

${ }^{\mathrm{qq}}$ Visitor from Office of Science, U.S. Department of Energy, Washington, DC 20585, USA.

${ }^{\mathrm{rr}}$ Visitor from Universidade Estadual Paulista, São Paulo, Brazil.

${ }^{\text {ss }}$ Visitor from Nagasaki Institute of Applied Science, Nagasaki 851-0193, Japan.

${ }^{\text {tt } V i s i t o r ~ f r o m ~ U n i v e r s i t e ~ C a t h o l i q u e ~ d e ~ L o u v a i n, ~} 1348$ Louvain-La-Neuve, Belgium.

${ }^{\mathrm{uu}}$ Visitor from University of California Irvine, Irvine, CA 92697, USA.

${ }^{\mathrm{vv}}$ Visitor from European Organization for Nuclear Research (CERN).

${ }^{w w}$ Visitor from University of Fukui, Fukui City, Fukui Prefecture, Japan 910-0017.

[1] G. Aad et al. (ATLAS Collaboration), Phys. Lett. B 716, 1 (2012).

[2] S. Chatrchyan et al. (CMS Collaboration), Phys. Lett. B 716, 30 (2012).

[3] S. Chatrchyan et al. (CMS Collaboration), J. High Energy Phys. 06 (2013) 081.

[4] G. Aad et al. (ATLAS Collaboration), Phys. Rev. D 90, 052004 (2014).

[5] G. Aad et al. (ATLAS Collaboration), Report No. ATLASCONF-2014-042, 2014.

[6] S. Chatrchyan et al. (CMS Collaboration), Phys. Lett. B 736, 64 (2014).

[7] G. Aad et al. (ATLAS Collaboration), Phys. Lett. B 726, 88 (2013).

[8] G. Aad et al. (ATLAS Collaboration), Report No. ATLASCONF-2013-034, 2013.

[9] G. Aad et al. (ATLAS Collaboration), Phys. Rev. D 91, 012006 (2015).

[10] S. Chatrchyan et al. (CMS Collaboration), Phys. Rev. D 89, 092007 (2014).

[11] S. Chatrchyan et al. (CMS Collaboration), Eur. Phys. J. C 74, 3076 (2014).

[12] G. Aad et al. (ATLAS Collaboration), Report No. ATLASCONF-2013-040, 2013.

[13] G. Aad et al. (ATLAS Collaboration), Phys. Lett. B 726, 120 (2013).

[14] S. Chatrchyan et al. (CMS Collaboration), Phys. Rev. Lett. 110, 081803 (2013).

[15] S. Chatrchyan et al. (CMS Collaboration), J. High Energy Phys. 01 (2014) 096.

[16] V. Khachatryan et al. (CMS Collaboration), arXiv: 1411.3441 .
[17] T. Aaltonen et al. (CDF and D0 Collaborations), Phys. Rev. Lett. 109, 071804 (2012).

[18] T. Aaltonen et al. (CDF and D0 Collaborations), Phys. Rev. D 88, 052014 (2013).

[19] T. Aaltonen et al. (CDF Collaboration), Phys. Rev. D 88, 052013 (2013).

[20] V. M. Abazov et al. (D0 Collaboration), Phys. Rev. D 88, 052011 (2013).

[21] T. Aaltonen et al. (CDF Collaboration), Phys. Rev. Lett. 114, 141802 (2015).

[22] V. M. Abazov et al. (D0 Collaboration), Phys. Rev. Lett. 113, 161802 (2014).

[23] J. Ellis, D. S. Hwang, V. Sanz, and T. You, J. High Energy Phys. 11 (2012) 134.

[24] D. J. Miller, S. Y. Choi, B. Eberle, M. M. Mühlleitner, and P. M. Zerwas, Phys. Lett. B 505, 149 (2001).

[25] S. Chatrchyan et al. (CMS Collaboration), Nat. Phys. 10, 557 (2014).

[26] S. Chatrchyan et al. (CMS Collaboration), J. High Energy Phys. 05 (2014) 104.

[27] G. Aad et al. (ATLAS Collaboration), J. High Energy Phys. 09 (2012) 070.

[28] G. Aad et al. (ATLAS Collaboration), arXiv:1501.04943 [J. High Energy Phys. (to be published)].

[29] S. Chatrchyan et al. (CMS Collaboration), Phys. Rev. D 89, 012003 (2014).

[30] G. Aad et al. (ATLAS Collaboration), J. High Energy Phys. 01 (2015) 069.

[31] T. Aaltonen et al. (CDF Collaboration), Phys. Rev. Lett. 109, 111804 (2012).

[32] V. M. Abazov et al. (D0 Collaboration), Phys. Rev. D 88, 052008 (2013).

[33] T. Aaltonen et al. (CDF Collaboration), Phys. Rev. Lett. 109, 111803 (2012).

[34] V. M. Abazov et al. (D0 Collaboration), Phys. Rev. D 88, 052010 (2013).

[35] T. Aaltonen et al. (CDF Collaboration), Phys. Rev. D 87, 052008 (2013).

[36] V. M. Abazov et al. (D0 Collaboration), Phys. Lett. B 716, 285 (2012).

[37] Positions and angles are expressed in a cylindrical coordinate system, with the $z$ axis directed along the proton beam. The polar angle $\theta$ is defined with respect to the proton beam direction. The missing transverse energy is defined as a sum over calorimeter towers $\vec{E}_{T}=-\sum_{i} E_{i} \sin \theta_{i} \hat{n}_{i}$, where $i$ is the calorimeter tower number, and $\hat{n}_{i}$ is a unit vector perpendicular to the beam axis and pointing to the $i$ th tower. The reconstructed $\vec{E}_{T}$ is corrected for contributions from muons, which register less energy in the calorimeter, and for jet energy corrections. The scalar magnitude of $\vec{E}_{T}$ is denoted $E_{T}$.

[38] D. Acosta et al. (CDF Collaboration), Phys. Rev. D 71, 032001 (2005); A. Abulencia et al. (CDF Collaboration), J. Phys. G 34, 2457 (2007).

[39] V. M. Abazov et al. (D0 Collaboration), Nucl. Instrum. Methods Phys. Res., Sect. A 565, 463 (2006); M. Abolins et al., Nucl. Instrum. Methods Phys. Res., Sect. A 584, 75 (2008); R. Angstadt et al., Nucl. Instrum. Methods Phys. Res., Sect. A 622, 298 (2010). 
[40] T. Sjöstrand, S. Mrenna, and P. Skands, J. High Energy Phys. 05 (2006) 026. We use PYTHIA version 6.216 to generate the Higgs boson signal events.

[41] H. L. Lai, J. Huston, S. Kuhlmann, F. I. Olness, J. F. Owens, D. E. Soper, W. K. Tung, and H. Weerts, Phys. Rev. D 55, 1280 (1997).

[42] J. Alwall, M. Herquet, F. Maltoni, O. Mattelaer, and T. Stelzer, J. High Energy Phys. 06 (2011) 128.

[43] J. Baglio and A. Djouadi, J. High Energy Phys. 10 (2010) 064.

[44] G. Ferrera, M. Grazzini, and F. Tramontano, Phys. Rev. Lett. 107, 152003 (2011).

[45] O. Brein, A. Djouadi, and R. Harlander, Phys. Lett. B 579, 149 (2004).

[46] M. L. Ciccolini, S. Dittmaier, and M. Kramer, Phys. Rev. D 68, 073003 (2003).

[47] S. Dittmaier et al. (LHC Higgs Cross Section Working Group), arXiv:1201.3084.

[48] A. Djouadi, J. Kalinowski, and M. Spira, Comput. Phys. Commun. 108, 56 (1998).

[49] A. Bredenstein, A. Denner, S. Dittmaier, and M. M. Weber, Phys. Rev. D 74, 013004 (2006); A. Bredenstein, A. Denner, S. Dittmaier, and M. M. Weber, J. High Energy Phys. 02 (2007) 080; A. Bredenstein, A. Denner, S. Dittmaier, A. Mück, and M. M. Weber, http://omnibus.uni-freiburg.de/ $\sim$ sd565/programs/prophecy4f/prophecy4f.html (2010).

[50] J. M. Campbell and R. K. Ellis, Phys. Rev. D 60, 113006 (1999).

[51] U. Langenfeld, S. Moch, and P. Uwer, Phys. Rev. D 80, 054009 (2009).

[52] T. Aaltonen et al. (CDF and D0 Collaborations), Phys. Rev. D 86, 092003 (2012).

[53] A. D. Martin, W. J. Stirling, R. S. Thorne, and G. Watt, Eur. Phys. J. C 63, 189 (2009).

[54] N. Kidonakis, Phys. Rev. D 74, 114012 (2006).

[55] In the CDF analyses, backgrounds from SM processes with electroweak gauge bosons or top quarks are modeled using PYTHIA [40], ALPGEN [56], MC@NLO [57], and HERWIG [58]. For D0, these backgrounds are modeled using PYTHIA, ALPGEN, and SINGLETOP [59], with PYTHIA providing parton showering and hadronization for all the generators.
[56] M. L. Mangano, M. Moretti, F. Piccinini, R. Pittau, and A. D. Polosa, J. High Energy Phys. 07 (2003) 001.

[57] S. Frixione and B. R. Webber, J. High Energy Phys. 06 (2002) 029.

[58] G. Corcella, I. G. Knowles, G. Marchesini, S. Moretti, K. Odagiri, P. Richardson, M. H. Seymour, and B. R. Webber, J. High Energy Phys. 01 (2001) 010.

[59] E. E. Boos, V. E. Bunichev, L. V. Dudko, V. I. Savrin, and V. V. Sherstnev, Phys. At. Nucl. 69, 1317 (2006).

[60] A heavy-flavor jet is a reconstructed cluster of calorimeter energies associated with particles produced in the hadronization and decay of a $b$ or $c$ quark.

[61] A $b$-tagged jet is a jet identified as being consistent with that expected from the decay products of a $b$ quark based on properties such as the presence of displaced track vertices or soft leptons.

[62] T. Aaltonen et al. (CDF Collaboration), Phys. Rev. Lett. 109, 111802 (2012).

[63] V. M. Abazov et al. (D0 Collaboration), Phys. Rev. Lett. 109, 121802 (2012).

[64] J. Freeman, T. Junk, M. Kirby, Y. Oksuzian, T. J. Phillips, F. D. Snider, M. Trovato, J. Vizan, and W. M. Yao, Nucl. Instrum. Methods Phys. Res., Sect. A 697, 64 (2013).

[65] V. M. Abazov et al. (D0 Collaboration), Nucl. Instrum. Methods Phys. Res., Sect. A 620, 490 (2010).

[66] V. M. Abazov et al. (D0 Collaboration), Nucl. Instrum. Methods Phys. Res., Sect. A 763, 290 (2014).

[67] T. Aaltonen et al. (CDF Collaboration), Phys. Rev. Lett. 105, 251802 (2010).

[68] L. Breiman, Mach. Learn. 45, 5 (2001).

[69] A. Hocker et al., Proc. Sci. ACAT2007 (2007) 040 [arXiv: physics/0703039].

[70] S. Klimenko, J. Konigsberg, and T. M. Liss, Report No. FERMILAB-FN-0741, 2003.

[71] T. Junk, Nucl. Instrum. Methods Phys. Res., Sect. A 434, 435 (1999); A. L. Read, J. Phys. G 28, 2693 (2002).

[72] W. Fisher, Report No. FERMILAB-TM-2386-E, 2006.

[73] See the Supplemental Material at http://link.aps.org/ supplemental/10.1103/PhysRevLett.114.151802 for additional figures.

[74] ATLAS and CMS Collaborations, Report No. ATLASPHYS-PUB-2011-011, CMS NOTE-2011/005, 2011. 